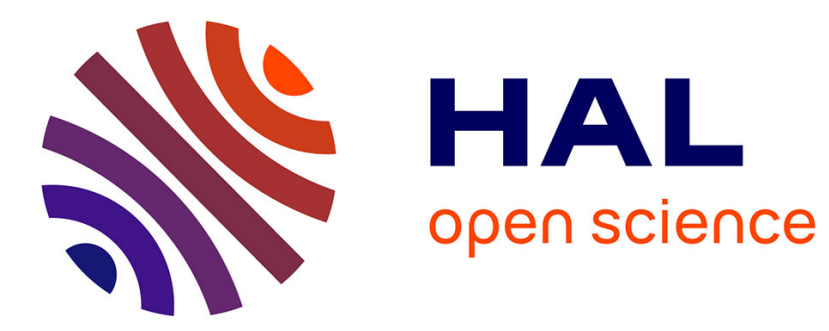

\title{
Inter-phase heat transfer and energy coupling in turbulent dispersed multiphase flows
}

\author{
Y. Ling, S. Balachandar, M. Parmar
}

\section{To cite this version:}

Y. Ling, S. Balachandar, M. Parmar. Inter-phase heat transfer and energy coupling in turbulent dispersed multiphase flows. Physics of Fluids, 2016, 28, pp.033304. 10.1063/1.4942184 . hal-01311859

\section{HAL Id: hal-01311859 \\ https://hal.sorbonne-universite.fr/hal-01311859}

Submitted on 4 May 2016

HAL is a multi-disciplinary open access archive for the deposit and dissemination of scientific research documents, whether they are published or not. The documents may come from teaching and research institutions in France or abroad, or from public or private research centers.
L'archive ouverte pluridisciplinaire HAL, est destinée au dépôt et à la diffusion de documents scientifiques de niveau recherche, publiés ou non, émanant des établissements d'enseignement et de recherche français ou étrangers, des laboratoires publics ou privés. 


\section{AIP $\left.\right|_{\text {Physics of }}$ Fluids}

\section{Inter-phase heat transfer and energy coupling in turbulent dispersed multiphase flows}

Y. Ling, S. Balachandar, and M. Parmar

Citation: Physics of Fluids 28, 033304 (2016); doi: 10.1063/1.4942184

View online: http://dx.doi.org/10.1063/1.4942184

View Table of Contents: http://scitation.aip.org/content/aip/journal/pof2/28/3?ver=pdfcov

Published by the AIP Publishing

\section{Articles you may be interested in}

Turbulent concentration diffusion in multiphase flow

Phys. Fluids 24, 093301 (2012); 10.1063/1.4748348

Axial dispersion in segmented gas-liquid flow: Effects of alternating channel curvature

Phys. Fluids 22, 122106 (2010); 10.1063/1.3531742

A Coupled Multiphase Fluid Flow And Heat And Vapor Transport Model For Air-Gap Membrane Distillation

AIP Conf. Proc. 1254, 109 (2010); 10.1063/1.3453795

Two-phase Couette-Taylor flow: Arrangement of the dispersed phase and effects on the flow structures

Phys. Fluids 16, 128 (2004); 10.1063/1.1630323

Linear instability of a particle-laden mixing layer with a dynamic dispersed phase

Phys. Fluids 10, 2539 (1998); 10.1063/1.869769

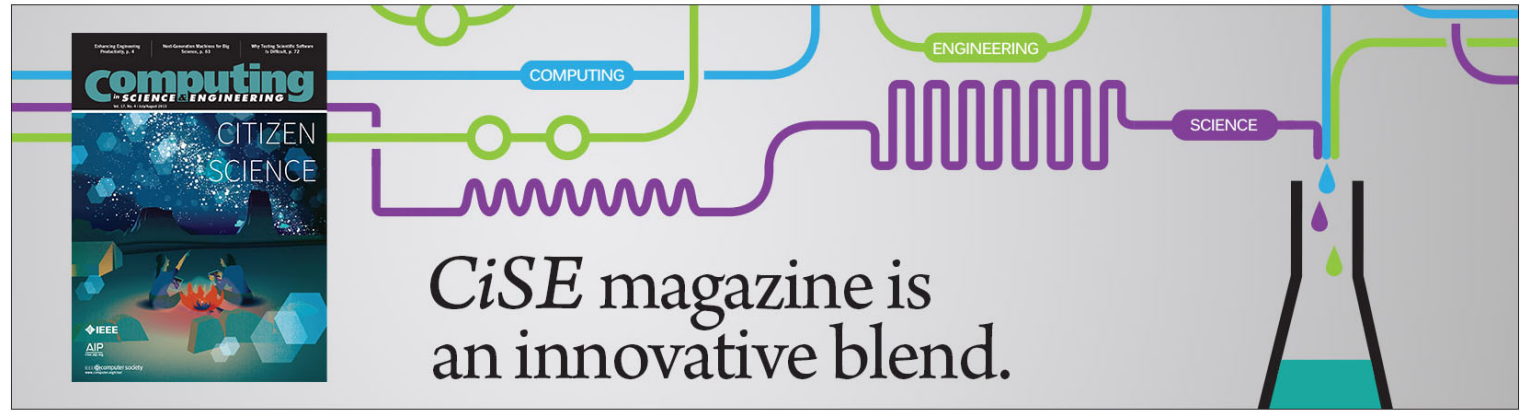




\title{
Inter-phase heat transfer and energy coupling in turbulent dispersed multiphase flows
}

\author{
Y. Ling, ${ }^{1, a)}$ S. Balachandar, ${ }^{2}$ and M. Parmar ${ }^{2}$ \\ ${ }^{1}$ Institut Jean Le Rond d'Alembert, Sorbonne Universités, UPMC Univ Paris 06, CNRS, UMR \\ 7190, Paris F-75005, France \\ ${ }^{2}$ Department of Mechanical and Aerospace Engineering, University of Florida, Gainesville, \\ Florida 32611, USA
}

(Received 4 September 2015; accepted 5 February 2016; published online 9 March 2016)

\begin{abstract}
The present paper addresses important fundamental issues of inter-phase heat transfer and energy coupling in turbulent dispersed multiphase flows through scaling analysis. In typical point-particle or two-fluid approaches, the fluid motion and convective heat transfer at the particle scale are not resolved and the momentum and energy coupling between fluid and particles are provided by proper closure models. By examining the kinetic energy transfer due to the coupling forces from the macroscale to microscale fluid motion, closure models are obtained for the contributions of the coupling forces to the energy coupling. Due to the inviscid origin of the added-mass force, its contribution to the microscale kinetic energy does not contribute to dissipative transfer to fluid internal energy as was done by the quasi-steady force. Time scale analysis shows that when the particle is larger than a critical diameter, the diffusive-unsteady kernel decays at a time scale that is smaller than the Kolmogorov time scale. As a result, the computationally costly Basset-like integral form of diffusive-unsteady heat transfer can be simplified to a non-integral form. Conventionally, the fluid-toparticle volumetric heat capacity ratio is used to evaluate the relative importance of the unsteady heat transfer to the energy balance of the particles. Therefore, for gas-particle flows, where the fluid-to-particle volumetric heat capacity ratio is small, unsteady heat transfer is usually ignored. However, the present scaling analysis shows that for small fluid-to-particle volumetric heat capacity ratio, the importance of the unsteady heat transfer actually depends on the ratio between the particle size and the Kolmogorov scale. Furthermore, the particle mass loading multiplied by the heat capacity ratio is usually used to estimate the importance of the thermal two-way coupling effect. Through scaling argument, improved estimates are established for the energy coupling parameters of each energy exchange mechanism between the phases. (C) 2016 AIP Publishing LLC. [http://dx.doi.org/10.1063/1.4942184]
\end{abstract}

\section{INTRODUCTION}

Inter-phase heat transfer and energy coupling are of essential importance to many applications of turbulent dispersed multiphase flows. The dispersed multiphase flows of interest in the present study consist of a "carrier phase" and a "dispersed phase." The latter can be in the form of discrete elements like solid particles, droplets, and bubbles. For convenience of discussion, here after we refer to the dispersed and carrier phases as "particle" and "fluid," but it should be noted that the terms "particle" and "fluid" are used in a broad sense.

Numerical simulation is an important approach to investigate turbulent dispersed multiphase flows. In many practical applications, the number of particles is huge and the scales of primary interest are much larger than the size of individual particles. Therefore, the point-particle approach

\footnotetext{
a)Electronic mail: yueling@ dalembert.upmc.edu.
} 
(PPA) is commonly used, in which the particles are modeled as point masses. Since the flows around the particles are not resolved, the momentum and energy coupling between fluid and particles are represented by proper closure models.

To accurately represent the momentum coupling between the two phases, it is essential to rigorously model the force on each individual particle due to fluid-particle interaction. The overall force exerted on a spherical particle undergoing arbitrary time-dependent motion in an unsteady inhomogeneous ambient flow can be derived analytically in the limit of zero Reynolds and Mach numbers. ${ }^{1-4}$ Extension of the force model to finite Reynolds and Mach numbers unavoidably involves empirical correlations. ${ }^{5,6}$ The overall inter-phase coupling force can be separated into different physically meaningful contributions: the quasi-steady force $\mathbf{F}_{q s}$, the stress-gradient force $\mathbf{F}_{s g}$, the added-mass force $\mathbf{F}_{a m}$, and the viscous-unsteady force $\mathbf{F}_{v u}$ (often called the Basset history force). The latter three contributions together are loosely referred as the "unsteady forces," since they are non-zero only when the acceleration of the fluid or the particle is non-zero. Details of the inter-phase momentum coupling and the modeling of coupling force can be found in the work of Balachandar, ${ }^{7}$ Balachandar and Eaton, ${ }^{8}$ and Ling et al.,${ }^{9}$ and thus are not repeated here.

Similarly, the inter-phase energy coupling is represented by the inter-phase heat transfer model. The heat transfer between a spherical particle and the ambient fluid in an unsteady inhomogeneous flow can also be derived in the limit of zero Reynolds number. ${ }^{10}$ The overall inter-phase heat transfer can again be separated into physically meaningful contributions: the quasi-steady heat transfer $Q_{q s}$, the undisturbed-unsteady heat transfer $Q_{u u}$, and the diffusive-unsteady heat transfer $Q_{d u}$, which are the thermal analogues of $\mathbf{F}_{q s}, \mathbf{F}_{s g}$, and $\mathbf{F}_{v u}$. Note that there is no thermal analog to $\mathbf{F}_{a m}$ because the latter is inviscid in nature and arises from the no-penetration boundary condition. Again, the latter two contributions to inter-phase heat transfer together are loosely referred as the "unsteady heat transfer," since they are non-zero only when the thermal acceleration of the fluid or the particle is non-zero. The extensions of the inter-phase heat transfer model to finite Reynolds number were presented by Feng and Michaelides ${ }^{11}$ and Balachandar and Ha. ${ }^{12}$ Apart from heat transfer between the fluid and the particles, the inter-phase coupling forces can also contribute to the energy coupling between the two phases through the work done by the inter-phase coupling force. For example, the back effect of the quasi-steady force on the fluid contributes to dissipative heating of the ambient fluid. The energy contribution from inter-phase force-coupling to the fluid thermal field is generally small; however, it can become significant in some applications. ${ }^{13}$

Recently, scaling analysis of the inter-phase momentum coupling in turbulent dispersed multiphase flows was reported by Ling et al. ${ }^{14}$ By analyzing the time scales of turbulent flows and particle motion, useful estimates are obtained on the importance of unsteady forces compared to the quasi-steady force, and on the importance of the inter-phase coupling forces in influencing the ambient flow momentum balance. In this paper, we will extend the scaling analysis of Ling et al. ${ }^{14}$ to investigate inter-phase heat transfer and energy coupling. In particular, we are interested in addressing the following four fundamental questions regarding inter-phase energy coupling in turbulent dispersed multiphase flows:

- Q1: How do different inter-phase coupling forces contribute to inter-phase energy coupling and what are the correct formulations?

- Q2: Is it possible to simplify the history integral in computing the diffusive-unsteady heat transfer? If so, under what conditions can this simplification be made?

- Q3: Under what conditions is unsteady heat transfer important in evaluating the particle temperature evolution, when compared to the quasi-steady heat transfer?

- Q4: Under what conditions the back effects of the inter-phase heat transfer and coupling force need to be taken into account in the fluid energy equation (i.e., under what conditions the fluid and the particles are considered thermally two-way coupled)?

For Q1, it is generally considered that the discrepancy between the work done by the inter-phase coupling force on the fluid (e.g., $\mathbf{F}_{q s} \cdot \mathbf{u}_{f}$ ) and on the particles (e.g., $-\mathbf{F}_{q s} \cdot \mathbf{u}_{p}$ ) is transferred to the kinetic energy of the microscale fluid motion (at the particle scale), which will be eventually transferred to the internal energy of the ambient fluid due to dissipation. This argument is valid for the quasi-steady force due to its viscous nature. Similarly the contribution of the work done by 
the unsteady forces to the internal energy of the carrier phase must be clarified. Here we consider $\mathbf{u}_{f}$ is the instantaneous undisturbed fluid velocity at the particle location. If the mean value is used instead, then it has been shown recently by Xu and Subramaniam ${ }^{15}$ and Mehrabadi et al. ${ }^{16}$ that the work done by the inter-phase coupling force can indeed contribute to kinetic energy transfer between the mean flow and the fluctuating motion of fluid and particles.

For Q2, the diffusive-unsteady heat transfer is generally computed as the Basset-like convolution integral of the past history of relative thermal acceleration between the particle and the surrounding fluid weighted by the history kernel. ${ }^{10}$ The evaluation of this convolution integral can be computationally expensive if must be carried out for a large number of particles. If the rate of change of relative thermal acceleration is slower than the decay of the thermal history kernel then the convolution integral can be simplified and precomputed. ${ }^{12}$ In this paper, by investigating the time scale of the diffusive-unsteady heat transfer kernel in relation to the turbulence time scales, we will establish the condition under which the convolution integral can be simplified.

For Q3, the importance of unsteady forces in momentum coupling is usually estimated in terms of the fluid-to-particle density ratio $\rho_{f} / \rho_{p}$. Similarly, the fluid-to-particle volumetric heat capacity ratio $\beta_{\theta}=\rho_{f} C_{f} / \rho_{p} C_{p}$ can be used to assess the importance of unsteady heat transfer, where $C_{p}$ and $C_{f}$ are the specific heat capacities of the particle and the fluid. (Here the heat capacity of fluid corresponds to that at constant volume.) Through scaling estimates of relative velocity and relative acceleration for particles in turbulent flows, Ling et al. ${ }^{14}$ have established the relative importance of unsteady forces in three regimes characterized by $\tau_{p m}<\tau_{\eta}, \tau_{\eta}<\tau_{p m}<\tau_{L}$, and $\tau_{p m}>\tau_{L}$, where $\tau_{p m}$ is the particle mechanical response time and $\tau_{\eta}$ and $\tau_{L}$ are the Kolmogorov and integral time scales of the ambient flow. Following this line of reasoning, quantitative estimates of the relative importance of unsteady heat transfer compared to the quasi-steady heat transfer will be obtained in these three regimes of turbulent multiphase flows.

For Q4, the particle-to-fluid mass fraction ratio (or the particle mass fraction alone) is usually used as the momentum coupling parameter to assess the importance of back effect of the inter-phase coupling force on the fluid motion. ${ }^{17}$ When the particle-to-fluid mass fraction ratio is $O(1)$, the two phases are considered to be two-way mechanically coupled. The thermal extension of this conventional criterion would be the particle-to-fluid mass fraction ratio multiplied by the specific heat ratio. Nevertheless, it has been shown that the conventional momentum coupling parameter is valid only for the quasi-steady force but not for the unsteady forces. As a result, it is of interest to investigate the energy coupling parameters and evaluate under what conditions the two phases need to be considered thermally two-way coupled.

To simplify the analysis and focus on answering the above questions, several assumptions and approximations are made:

- The effect of temperature variation on the transport properties of the fluid is ignored.

- The effect of gravity is neglected and as a result there will be no buoyancy induced fluid motion.

- The Prandtl number of the fluid, $\operatorname{Pr}_{f}$, is close to unity. As a result, the inertial-diffusive subrange is negligibly small and the inertial-convective subrange dominates the spectra of temperature fluctuation.

- The particle Biot number is taken to be much smaller than unity and thus the temperature within the particle is uniform.

- There is no phase change or mass transfer between the particle and the fluid.

- The multiphase flow is in the dilute regime and therefore inter-particle interactions are negligible.

In the inertial-convective subrange, the effect of viscosity and thermal diffusivity is negligible. As a result, the spectral density function of the variance of the temperature fluctuation is shown to have the same decay rate as that of the turbulent kinetic energy, i.e., $k^{-5 / 3}$, where $k$ is the wavenumber. ${ }^{18,19}$ The Kolmogorov length scale for turbulent motion and temperature fluctuation is defined as $\eta_{m}=\left(v_{f}^{3} / \epsilon\right)^{1 / 4}$ and $\eta_{\theta}=\left(\alpha_{f}^{3} / \epsilon\right)^{1 / 4}$, where $v_{f}$ and $\alpha_{f}$ are the fluid viscous and thermal diffusivity, respectively. Since the fluid Prandtl number is considered to be close to one, then $\eta_{m} \approx \eta_{\theta}$. 
The governing equations for both the fluid and the particles and the models for the inter-phase coupling forces and heat transfer will be first described in Section II. In Section II E, we will discuss the correct expression of work done by each inter-phase coupling force in the fluid energy equation. A general analysis of particle and turbulent flow time scales is presented in Section III. Based on the estimates of these time scales, we will discuss the integral and non-integral representations of diffusive-unsteady heat transfer in Section IV. Scaling analysis of the inter-phase energy coupling is conducted in Section V. We will first evaluate the importance of unsteady heat transfer compared to quasi-steady heat transfer in Section V A. Then similar analysis will be done to investigate the importance of the contributions of inter-phase heat transfer and coupling force in backward coupling in Sections V B and V C, respectively. Finally, conclusions are drawn in Section VI.

\section{GOVERNING EQUATIONS}

\section{A. PPA}

The point-particle approach is a useful method in the simulation of dispersed multiphase flows, including inviscid ${ }^{20}$ and viscous (turbulent) flows, ${ }^{21,22}$ incompressible $^{23,24}$ and compressible flows. ${ }^{13,25,26}$ Here we pursue PPA formulated under the Eulerian-Lagrangian framework: the fluid is viewed as a continuum and the governing equations are written in the Eulerian framework, while the particles are retained as discrete point masses and tracked in the Lagrangian framework. The governing equations for the fluid and the particles can be written in different ways. The following set of equations is expressed in a manner well suited for the present Eulerian-Lagrangian framework. The equivalence of this set of governing equations to more conventional form used in two-fluid approaches will be discussed later,

$$
\begin{aligned}
\frac{\partial\left(\rho_{f} \phi_{f}\right)}{\partial t}+\nabla \cdot\left(\rho_{f} \phi_{f} \mathbf{u}_{f}\right) & =0 \\
\rho_{f} \phi_{f} \frac{D \mathbf{u}_{f}}{D t}-\nabla \cdot \sigma_{f} & =-\frac{1}{\mathbb{V}} \sum_{i}^{N_{p}} \mathbf{F}_{f p, i}, \\
\rho_{f} \phi_{f} \frac{D E_{f}}{D t}-\nabla \cdot\left(\sigma_{f} \cdot\left(\phi_{f} \mathbf{u}_{f}+\phi_{p} \mathbf{u}_{p}\right)\right)-\nabla \cdot \mathbf{q}_{f} & =-\frac{1}{\mathbb{V}} \sum_{i}^{N_{p}}\left(G_{f p, i}+Q_{f p, i}\right),
\end{aligned}
$$

and

$$
\begin{aligned}
\frac{d \mathbf{x}_{p, i}}{d t} & =\mathbf{u}_{p, i}, \\
m_{p} \frac{d \mathbf{u}_{p, i}}{d t} & =\mathbf{F}_{f p, i}, \\
m_{p} C_{s} \frac{d T_{p, i}}{d t} & =Q_{f p, i} .
\end{aligned}
$$

The subscripts $f$ and $p$ denote properties associated with the fluid and particles, respectively. The fluid variables, $\rho_{f}, \phi_{f}$, and $E_{f}$, represent the density, volume fraction, and total macroscale energy of fluid. The bold symbols $\mathbf{u}_{f}, \sigma_{f}$, and $\mathbf{q}_{f}$ represent the velocity vector, stress tensor, and heat flux vector of fluid. The variable $\mathbf{u}_{p}$ appearing in Eq. (3) represent the average particle velocity and detailed discussion of which is given in Section II D.

The position and velocity vectors and temperature of the $i$ th particle are denoted by $\mathbf{x}_{p, i}, \mathbf{u}_{p, i}$, and $T_{p, i}$. For simplicity the mass and heat capacity of the particles are taken to be constants $m_{p}$ and $C_{p}$. The overall force on the $i$ th particle due to fluid-particle coupling is denoted by $\mathbf{F}_{f p, i}$, while the overall convective heat transfer between the fluid and particles is denoted by $Q_{f p, i}$.

In PPA, as the flow around particles is not resolved, the expressions of $\mathbf{F}_{f p, i}$ and $Q_{f p, i}$ are given by fluid-particle coupling models in terms of the macroscale (undisturbed) fluid flow properties. To conserve momentum and energy of the whole system, $\mathbf{F}_{f p, i}$ and $Q_{f p, i}$ need to be subtracted from the fluid momentum and energy equations (Eqs. (2) and (3)) to account for the back effect of the particles on the fluid motion and temperature evolution. In Eq. (3), $G_{f p, i}$ corresponds to 
contribution to the total energy of the fluid from the inter-phase coupling force $\mathbf{F}_{f p, i}$. In Eqs. (2) and (3), $(1 / \mathbb{V}) \sum_{i}^{N_{p}}$ symbolically represent the net feedback from the particles onto the carrier phase. In a finite volume context, this feedback is expressed as a sum over all the particles within the cell, where $N_{p}$ is the total number of particles in the cell and $\mathbb{V}$ denotes the cell volume. If needed the feedback can be computed in more sophisticated ways, ${ }^{27}$ but the form given in Eqs. (2) and (3) is sufficient for the present scaling analysis.

\section{B. Inter-phase coupling force}

For an individual particle, the equation of motion, Eq. (5), can be written as

$$
m_{p, i} \frac{d \mathbf{u}_{p, i}}{d t}=\mathbf{F}_{f p, i}=\mathbf{F}_{s g, i}+\mathbf{F}_{q s, i}+\mathbf{F}_{a m, i}+\mathbf{F}_{v u, i},
$$

where $\mathbf{F}_{s g, i}, \mathbf{F}_{q s, i}, \mathbf{F}_{a m, i}$, and $\mathbf{F}_{v u, i}$ represent the stress-gradient, quasi-steady, added-mass, and viscous-unsteady forces. The expressions of these forces are given as

$$
\begin{aligned}
\mathbf{F}_{s g, i} & =V_{p, i}\left(\nabla \cdot \boldsymbol{\sigma}_{f}\right)_{i}, \\
\mathbf{F}_{q s, i} & =3 \pi \mu_{f, i} d_{p, i}\left(\mathbf{u}_{f, i}-\mathbf{u}_{p, i}\right) \Phi\left(\operatorname{Re}_{p, i}\right), \\
\mathbf{F}_{a m, i} & =V_{p, i} C_{M}\left(\nabla \cdot \sigma_{f}-\frac{d \rho_{f} \mathbf{u}_{p}}{d t}\right)_{i} \\
\mathbf{F}_{v u, i} & =\frac{3}{2} d_{p, i}^{2} \sqrt{\pi v_{f, i}} \sqrt{\tau_{v u, i}} \int_{-\infty}^{t} K_{v u}\left(\frac{t-\xi}{\tau_{v u, i}}, \operatorname{Re}_{p, i}\right)\left(\nabla \cdot \boldsymbol{\sigma}_{f}-\frac{d \rho_{f} \mathbf{u}_{p}}{d t}\right)_{i} \frac{d \xi}{\tau_{v u, i}},
\end{aligned}
$$

where $V_{p}$ and $d_{p}$ denote the particle volume and diameter. The variables with a subscript ${ }_{f, i}$ represent the fluid variables at the $i$ th particle location, e.g., $\mu_{f, i}$ represents the fluid dynamic viscosity at $\mathbf{x}_{p, i}$. The stress gradient force is the force due to the undisturbed ambient flow (i.e., due to the incident flow without being modified by the particle). In the quasi-steady force formulation, the correction function that accounts for the effect of finite Reynolds number is denoted by Ф. Empirical correlations of this correction function are given in Refs. 28 and 29. The particle Reynolds number, $\operatorname{Re}_{p}$, is defined based on the relative velocity between the fluid and the particle as $\operatorname{Re}_{p}=\left|\mathbf{u}_{f}-\mathbf{u}_{p}\right| d_{p} / v_{f}$. (For expressions that are identically applied to every point-particle, such as the one for $\operatorname{Re}_{p}$ here, the subscript $i$ is dropped here for conciseness.) The viscous-unsteady kernel, $K_{v u}$, is defined as a function of normalized time scaled by the viscous-unsteady time scale, $\tau_{v u}$. The detailed expressions of $K_{v u}$ and $\tau_{v u}$ are given by Mei and Adrian ${ }^{5}$ and Ling et al. ${ }^{14}$

In Eqs. (10) and (11), the term $\nabla \cdot \sigma_{f}$ is often replaced by $\rho_{f} D \mathbf{u}_{f} / D t^{1}$ and $D \rho_{f} \mathbf{u}_{f} / D t^{4}$ in incompressible and compressible flows, respectively. It has been argued that the first two forms are identically equal according to the Navier-Stokes equations of the fluid phase. This is indeed true when one considers force on an isolated particle immersed in a fluid flow, where quantities such as $\mathbf{u}_{f}$ and $\rho_{f}$ correspond to those of the pure fluid. However, in a two-way coupled multiphase system, as can be readily seen from Eq. (2), the different forms are not equivalent. In Eqs. (1)-(3), $\mathbf{u}_{f}$ and other fluid phase quantities are at the macroscale and must be interpreted as spatial or ensemble averages. It remains somewhat unclear as to which form should be used in Eqs. (10) and (11). Nevertheless, the results to be obtained by the scaling analysis in the present work are independent of this detail.

\section{Inter-phase coupling heat transfer}

The equation of the thermal evolution of an individual particle can be written as

$$
m_{p} C_{p} \frac{d T_{p}}{d t}=Q_{f p}=Q_{u u}+Q_{q s}+Q_{d u},
$$

where $Q_{u u}, Q_{q s}$, and $Q_{d u}$ represent the undisturbed-unsteady, quasi-steady, and diffusive-unsteady contributions to the overall heat transfer due to inter-phase coupling. ${ }^{30}$ Their expressions are given as

$$
Q_{u u}=V_{p} \nabla \cdot \mathbf{q}_{f},
$$




$$
\begin{aligned}
& Q_{q s}=2 \pi \kappa_{f} d_{p}\left(T_{f}-T_{p}\right) \Psi\left(\operatorname{Re}_{p}, \operatorname{Pr}_{f}\right), \\
& Q_{d u}=\pi d_{p} \kappa_{f} \tau_{d u} \mathrm{Pe}_{p} \int_{-\infty}^{t} K_{d u}\left(\frac{t-\xi}{\tau_{d u}}, \mathrm{Pe}_{p}\right)\left(\frac{1}{\rho_{f} C_{f}} \nabla \cdot \mathbf{q}_{f}-\frac{d T_{p}}{d t}\right) \frac{d \xi}{\tau_{d u}},
\end{aligned}
$$

where $\kappa_{f}$ is the thermal conductivity of fluid. The temperature of fluid and particle is represented by $T_{f}$ and $T_{p}$, respectively. Here again in Eqs. (13) and (15), $\nabla \cdot \mathbf{q}_{f}$, which accounts for the undisturbed ambient thermal field seen by the particle, can be replaced by $\rho_{f} C_{f} D T_{f} / D t$, assuming the work induced heating is negligible. ${ }^{10}$

The first contribution, $Q_{u u}$, is analogous to the stress-gradient force $\mathbf{F}_{s g}$. It accounts for the heat transfer to the particle due to the undisturbed ambient flow field. On the right hand side of Eq. (12), the second contribution $Q_{q s}$ accounts for the quasi-steady thermal transfer from the particle to the surrounding fluid due to the instantaneous temperature difference. The correction function that accounts for the effect of finite Reynolds number on the quasi-steady heat transfer is denoted by $\Psi=\mathrm{Nu} / 2$, where $\mathrm{Nu}=\alpha_{f} d_{p} / \kappa_{f}$ is the Nusselt number and it is a function $\operatorname{Re}_{p}$ and $\operatorname{Pr}_{f}$. At the Stokes limit, $\mathrm{Nu}=2$ and $\Psi=1$. At finite Reynolds numbers, $\Psi$ is given by empirical correlations, see, e.g., Whitaker ${ }^{31}$ and Ranz and Marshall. ${ }^{32}$

The last contribution $Q_{d u}$ accounts for the unsteady thermal diffusion due to the temporal development of the thermal boundary layer around the particle and depends on the past history of relative thermal acceleration. The diffusive-unsteady kernel, $K_{d u}$, is defined as a function of time normalized by the diffusive-unsteady time scale, $\tau_{d u}$. It is noted that the diffusive-unsteady kernel $K_{d u}$ and time scale $\tau_{d u}$ are the thermal analogues of the viscous-unsteady kernel $K_{v u}$ and time scale $\tau_{v u}$, respectively. ${ }^{14}$ The diffusive-unsteady kernel generally depends on the particle Peclet number, which is defined as $\operatorname{Pe}_{p}=\operatorname{Re}_{p} \operatorname{Pr}_{f}$. The expression for $K_{d u}$ in incompressible flows in the zero Peclet (Reynolds) number limit is given by Michaelides and Feng. ${ }^{10}$ The extensions of $K_{d u}$ to finite Peclet (Reynolds) number have been presented by Feng and Michaelides ${ }^{11}$ and Balachandar and Ha. ${ }^{12}$

Under extreme conditions as in multiphase detonation, the solid particles can experience significant deformation. ${ }^{9}$ In such cases, compression heating and expansion cooling of the deforming particles will need to be considered in inter-phase energy coupling, but these effects are out of the scope of the present paper.

\section{Equivalence to the Eulerian-Eulerian (two-fluid) formulation}

As pointed out earlier, Eqs. (4)-(15) have been written in the Lagrangian framework as applied to each individual particle, and the gas governing equations in (1)-(3) have been expressed accordingly. Here we will consider the fluid and the particle phase governing equations as typically expressed in the Eulerian-Eulerian framework (or two-fluid formulation), ${ }^{17,33}$ so their equivalence can be established. In the two-fluid formulation, the fluid-phase governing equations can be expressed as

$$
\begin{array}{r}
\frac{\partial\left(\rho_{f} \phi_{f}\right)}{\partial t}+\nabla \cdot\left(\rho_{f} \phi_{f} \mathbf{u}_{f}\right)=0, \\
\frac{\partial\left(\rho_{f} \phi_{f} \mathbf{u}_{f}\right)}{\partial t}+\nabla \cdot\left(\rho_{f} \phi_{f} \mathbf{u}_{f} \mathbf{u}_{f}\right)-\nabla \cdot\left(\phi_{f} \boldsymbol{\sigma}_{f}\right)+\boldsymbol{\sigma}_{f} \cdot \nabla \phi_{f}= \\
-\rho_{p} \phi_{p}\left(\mathbf{f}_{q s}+\mathbf{f}_{a m}+\mathbf{f}_{v u}\right), \\
\frac{\partial\left(\rho_{f} \phi_{f} E_{f}\right)}{\partial t}+\nabla \cdot\left(\rho_{f} \phi_{f} \mathbf{u}_{f} E_{f}\right)-\nabla \cdot\left(\phi_{f} \boldsymbol{\sigma}_{f} \cdot \mathbf{u}_{f}\right)-\phi_{f} \nabla \cdot \mathbf{q}_{f}= \\
\sigma_{f}: \nabla\left(\phi_{p} \mathbf{u}_{p}\right)-\rho_{p} \phi_{p}\left(g_{q s}+g_{a m}+g_{v u}\right)-\rho_{p} \phi_{p}\left(q_{q s}+q_{d u}\right) .
\end{array}
$$

The particle phase properties, such as velocity $\mathbf{u}_{p}$ and total energy $E_{p}$, must be interpreted as field variables in the two-fluid formulation. The corresponding particle phase governing equations now mirror those of the gas phase and can be expressed as

$$
\frac{\partial\left(\rho_{p} \phi_{p}\right)}{\partial t}+\nabla \cdot\left(\rho_{p} \phi_{p} \mathbf{u}_{p}\right)=0
$$




$$
\begin{array}{r}
\frac{\partial\left(\rho_{p} \phi_{p} \mathbf{u}_{p}\right)}{\partial t}+\nabla \cdot\left(\rho_{p} \phi_{p} \mathbf{u}_{p} \mathbf{u}_{p}\right)-\nabla \cdot\left(\phi_{p} \boldsymbol{\sigma}_{f}\right)+\sigma_{f} \cdot \nabla \phi_{p}= \\
\rho_{p} \phi_{p}\left(\mathbf{f}_{q s}+\mathbf{f}_{a m}+\mathbf{f}_{v u}\right), \\
\frac{\partial\left(\rho_{p} \phi_{p} E_{p}\right)}{\partial t}+\nabla \cdot\left(\rho_{p} \phi_{p} \mathbf{u}_{p} E_{p}\right)-\nabla \cdot\left(\phi_{p} \boldsymbol{\sigma}_{f} \cdot \mathbf{u}_{p}\right)-\phi_{p} \nabla \cdot \mathbf{q}_{f}= \\
-\sigma_{f}: \nabla\left(\phi_{p} \mathbf{u}_{p}\right)+\rho_{p} \phi_{p}\left(f_{q s}+f_{a m}+f_{v u}\right) \cdot \mathbf{u}_{p}+\rho_{p} \phi_{p}\left(q_{q s}+q_{d u}\right) .
\end{array}
$$

Several comments are given in order as follows with regard to the above equations as compared to governing equations (1)-(12) presented earlier. First, in the above two-fluid formulation, the left hand side of the equations are in the conservative form, except for the nozzling terms, $\sigma_{f} \cdot \nabla \phi_{f}$ and $\sigma_{f} \cdot \nabla \phi_{p}$, in the momentum equations and the heat conduction terms, $-\phi_{f} \nabla \cdot \mathbf{q}_{f}$ and $-\phi_{p} \nabla \cdot \mathbf{q}_{f}$, in the energy equations. The right hand sides of the momentum equations correspond to inter-phase exchange of momentum and by comparing (2) with (17) it can be observed,

$$
\mathbf{f}_{(s g, q s, a m, v u)}=\frac{1}{\rho_{p} \phi_{p} \mathbb{V}} \sum_{i}^{N_{p}} \mathbf{F}_{(s g, q s, a m, v u), i}
$$

Similarly from the energy equations we obtain

$$
g_{(s g, q s, a m, v u)}=\frac{1}{\rho_{p} \phi_{p} \mathbb{V}} \sum_{i}^{N_{p}} G_{(s g, q s, a m, v u), i}
$$

and

$$
q_{(u u, q s, d u)}=\frac{1}{\rho_{p} \phi_{p} \mathbb{V}} \sum_{i}^{N_{p}} Q_{(u u, q s, d u), i} .
$$

The right hand sides of the gas and the particle momentum equations add to zero, and thus the inter-phase exchange of momentum does not affect the global balance.

In the above equations, the total stress is the sum of pressure and the deviatoric (viscous) stress, i.e., $\boldsymbol{\sigma}_{f}=p_{f} \mathbf{I}+\boldsymbol{\tau}_{f}$, where $\mathbf{I}$ is the identity tensor. The pressure part of $\boldsymbol{\sigma}_{f} \cdot \nabla \phi_{f}$ in the fluid momentum (i.e., $p_{f} \nabla \phi_{f}$ ) can be identified as the inviscid nozzling force used in many two fluid models ${ }^{34}$ and its viscous generalization is the term $\sigma_{f} \cdot \nabla \phi_{f}$. The nozzling force does not appear explicitly in the fluid momentum equation given in Eq. (2). To reconcile, we first note that the contribution of the stress gradient force to the right hand side of Eq. (2) is given by $-\sum_{i}^{N_{p}} \mathbf{F}_{(s g), i} / \mathbb{V}$, where the sum is over all the particles within a grid cell. The stress gradient force on each particle is given by Eq. (10) and in a finite volume context if we take all the particles within a grid cell to experience the same stress gradient force, we obtain the sum to be equal to the local value of $\phi_{p} \nabla \cdot \sigma_{f}$. This stress gradient force combined with the stress divergence term, $-\nabla \cdot \sigma_{f}$, in Eq. (2) is identical to $-\nabla \cdot\left(\phi_{f} \sigma_{f}\right)+\sigma_{f} \cdot \nabla \phi_{f}$ in Eq. (17). Thus, the nozzling term is implicitly included in the momentum equation (Eq. (2)). Conversely, the stress gradient force does not explicitly appear in the two-fluid momentum equation (Eq. (17)).

In particle momentum equation (20), the third and the fourth terms on the left hand side combine to yield $\phi_{p} \nabla \cdot \sigma_{f}$ on the right hand side, which is nothing but the stress gradient force. Thus, it can be readily verified that Lagrangian particle momentum equation (5) when summed over all the particles within a grid cell is equivalent to the Eulerian version given in (20). The particle kinetic energy equation, obtained from dotting (20) with $\mathbf{u}_{p}$, can be subtracted from total energy equation (21) to obtain the following particle internal energy equation:

$$
\rho_{p} \phi_{p} C_{p} \frac{d T_{p}}{d t}=\phi_{p} \nabla \cdot \mathbf{q}_{f}+\rho_{p} \phi_{p}\left(q_{q s}+q_{d u}\right) .
$$

From (13) the first term on the right corresponds to heat transfer to the particle due to the undisturbed ambient flow. It can be seen that (25) is the same as Lagrangian energy equation (12) summed over all the particles with the grid cell.

We finally come to establishing the equivalence of the two forms of the fluid energy equations. We define the work done on the gas phase due to the stress gradient force to be 


$$
g_{s g}=\mathbf{f}_{s g} \cdot \mathbf{u}_{p} .
$$

This along with (23) and the definition of stress gradient force yield

$$
\frac{1}{\mathbb{V}} \sum_{i}^{N_{p}} \mathbf{G}_{s g}=\phi_{p}\left(\nabla \cdot \boldsymbol{\sigma}_{f}\right) \cdot \mathbf{u}_{p}
$$

It can be noted that the two-fluid energy equation given in (18) does not explicitly include either heat transfer due to the undisturbed ambient flow (i.e., $-\rho_{p} \phi_{p} q_{u u}$ ) or work due to the stress gradient force (i.e., $-\rho_{p} \phi_{p} g_{s g}$ ). Both these effects are due to the undisturbed flow and are only implicitly accounted for in (18). Using Eqs. (8), (13), and (27), these effects can be explicitly included on the right hand side of the energy equation. With additional manipulations and using relations (23) and (24), we can show that the continuous phase energy equation given in (3) is identical to that given in (18).

It should be reminded that in the two-fluid formulations, the spatial scale for averaging is considered to be smaller than the smallest flow length scale (e.g., Kolmogorov scale for turbulent flows). Therefore, Reynolds stress terms due to averaging of turbulent fluctuations do not appear.

\section{E. Fluid phase internal energy equation}

In (18) inter-phase coupling forces, $\mathbf{f}_{q s}, \mathbf{f}_{a m}$, and $\mathbf{f}_{v u}$, contribute to the total energy of the fluid through the rate of work terms $g_{q s}, g_{a m}$, and $g_{v u}$. The relations between the $g$ and the $\mathbf{f}$ terms can be expected to be analogous to that given in (26) for the stress gradient force. We first obtain the following internal energy equation for the fluid phase by subtracting the kinetic energy component from (18):

$$
\begin{aligned}
\rho_{f} \phi_{f} \frac{D e_{f}}{D t} & =\phi_{f} \nabla \cdot \mathbf{q}_{f}-\rho_{p} \phi_{p}\left(q_{q s}+q_{d u}\right)+\sigma_{f}: \nabla\left(\phi_{f} \mathbf{u}_{f}+\phi_{p} \mathbf{u}_{p}\right) \\
& +\rho_{p} \phi_{p}\left[\left(\mathbf{f}_{q s} \cdot \mathbf{u}_{f}-g_{q s}\right)+\left(\mathbf{f}_{a m} \cdot \mathbf{u}_{f}-g_{a m}\right)+\left(\mathbf{f}_{v u} \cdot \mathbf{u}_{f}-g_{v u}\right)\right] .
\end{aligned}
$$

The first two terms on the right are analogous to those in the particle phase internal energy equation given in (25). Summing the first term on the right of Eq. (25) and that of Eq. (28) yields total conductive heat transfer, i.e., $\nabla \cdot \mathbf{q}_{f}$, while the second terms of Eqs. (25) and (28) cancel each other, as they represent inter-phase heat transfer.

Based on the form of the third term on the right of Eq. (28), the following Newtonian constitutive model is often proposed for the deviatoric stress in a multiphase flow: 35

$$
\boldsymbol{\tau}_{f}=\sigma_{f}-p_{f} \mathbf{I}=\mu\left(\nabla \mathbf{u}_{m}+\left(\nabla \mathbf{u}_{m}\right)^{T}\right)+\left(\lambda-\frac{2}{3} \mu\right) \mathbf{I} \nabla \cdot \mathbf{u}_{m},
$$

where $\mu$ and $\lambda$ are the shear and bulk viscosities of the multiphase flow mixture (which can be substantially different from those of the pure fluid). The volume weighted mixture velocity is defined as

$$
\mathbf{u}_{m}=\phi_{f} \mathbf{u}_{f}+\phi_{p} \mathbf{u}_{p} .
$$

With the above constitutive relation we can see that the pressure part of the third term of Eq. (28) yields $-p \nabla \cdot \mathbf{u}_{m}$. This term corresponds to adiabatic compression heating or expansion cooling of the multiphase flow, and it depends on the divergence of the mixture velocity. In the above model, this reversible heating or cooling directly influences only the fluid internal energy and the contribution is generally small compared to heat transfer except for highly compressible flows. The contribution of the deviatoric part of the third term of Eq. (28), $\boldsymbol{\tau}_{f}: \nabla \mathbf{u}_{m}$, is guaranteed to be non-negative and corresponds to viscous dissipation. Again, the contribution of viscous dissipation to the fluid internal energy balance is generally small.

\section{F. Energy implications of inter-phase coupling forces}

Earlier we noted that in both point-particle and two-fluid approaches, $\mathbf{u}_{f}$ represents macroscale fluid motion at scales much larger than the particle size. Thus, fluid velocity and all other fluid 
properties in (1)-(3) and in (16)-(18) are averaged quantities that have been suitably volume or ensemble averaged. In the two-fluid equations, $\mathbf{u}_{p}$ and all other particle properties are also averaged macroscale quantities. At the microscale, i.e., at the scale of the particle size, we expect the fluid velocity to vary from being close to the particle velocity in a layer close to the particles to being close to the macroscale fluid velocity away from the particles. In point-particle and two-fluid approaches, such microscale motion is implicitly accounted in the momentum equation through the inter-phase coupling forces and in the energy equations through inter-phase heat transfer and energy contributions of the inter-phase coupling forces.

In Eq. (28) we now consider the first part of the fourth term, i.e., $\rho_{p} \phi_{p}\left(\mathbf{f}_{q s} \cdot \mathbf{u}_{f}-g_{q s}\right)$. This term arises from the difference in the contribution of the quasi-steady force to the fluid kinetic energy $\left(\rho_{p} \phi_{p} \mathbf{f}_{q s} \cdot \mathbf{u}_{f}\right)$ and to the particle phase kinetic energy $\left(\rho_{p} \phi_{p} g_{q s}\right)$. Their difference corresponds to the flow of energy to the microscale fluid motion around the particles arising from the velocity difference between the fluid and the particle phases. Due to the viscous origin of the quasi-steady force, this microscale kinetic energy in turn is dissipated and transferred to the internal energy of the fluid. The proper closure for $g_{q s}$ is then given by

$$
g_{q s}=\mathbf{f}_{q s} \cdot \mathbf{u}_{p} .
$$

The quasi-steady force $\mathbf{f}_{q s}$ is oriented in the direction of relative velocity, $\mathbf{u}_{f}-\mathbf{u}_{p}$, and therefore it is guaranteed that this irreversible transfer to the internal energy of the fluid through the microscale fluid motion is non-negative, i.e., $\mathbf{f}_{q s} \cdot\left(\mathbf{u}_{f}-\mathbf{u}_{p}\right) \geq 0$. The transfer of macroscale kinetic energy due to the quasi-steady force is shown schematically in Fig. 1.

The energetics of the added-mass force requires further consideration. The added-mass force arises from the kinetic energy change in the inviscid microscale flow around the particle, due to the relative acceleration of the particle. Thus the term $\rho_{p} \phi_{p}\left(\mathbf{f}_{a m} \cdot \mathbf{u}_{f}-\mathbf{g}_{a m}\right)$ in Eq. (28) corresponds to kinetic energy transfer to the inviscid microscale fluid motion. However, since its origin is in inviscid mechanism it is not dissipated by viscosity and does not contribute to dissipative transfer to internal energy of the fluid. Furthermore, this term can take both positive and negative values and accordingly energy can flow back and forth between the macroscale motion and the microscale inviscid perturbation (indicated by the bi-directional arrow in Fig. 1). This energy in microscale inviscid motion should not be transferred to internal energy, as was done for the quasi-steady force. Therefore, the proper closure for $g_{a m}$ is to make $\rho_{p} \phi_{p}\left(\mathbf{f}_{a m} \cdot \mathbf{u}_{f}-\mathbf{g}_{a m}\right)=0$ and thus

$$
g_{a m}=\mathbf{f}_{a m} \cdot \mathbf{u}_{f} .
$$

In compressible flows, the added-mass force involves a history integral as the relative acceleration of the particle generates compression and rarefaction waves that propagate away from the particle at finite acoustic speed (see the work of Parmar et al. ${ }^{36}$ ). If relative acceleration between the particle and the surrounding fluid occurs on a time scale comparable to the acoustic time scale, then part of the macroscale kinetic energy can be radiated to the far-field and irreversibly lost (similar to viscous dissipation). Even so, this loss of energy from the kinetic energy of the system should not contribute to the internal energy of the fluid. Furthermore, the form of the added-mass force used in Eq. (10) assumes the time scale of relative acceleration to be slower than the acoustic time scale, thus allowing the use of an effective added-mass coefficient, without the need for a history integral. In this limit there is no radiated loss of energy, the energy exchange between the macroand microscale fluid motion is fully conservative.

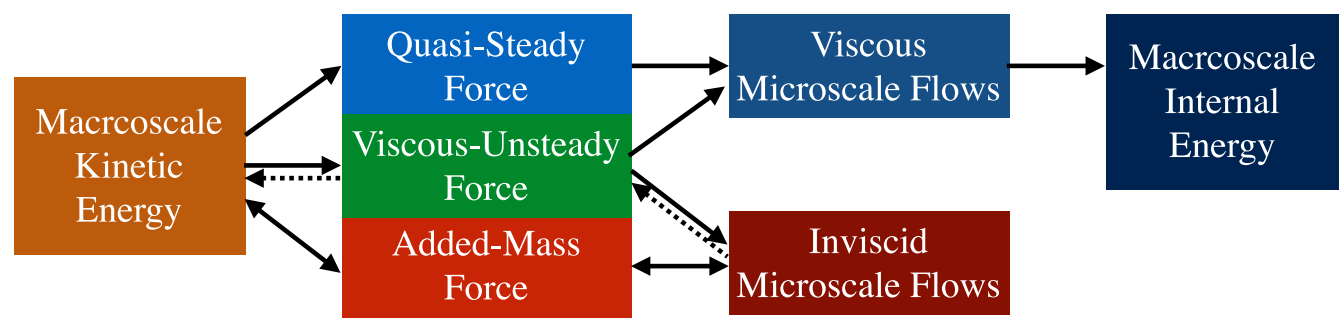

FIG. 1. Transfer of kinetic energy of the system due to the quasi-steady, added-mass, and viscous-unsteady forces. 
The kinetic energy transfer due to viscous-unsteady force is more complicated. Contribution to kinetic energy change in the macroscale system due to the viscous-unsteady force is partially dissipative like the quasi-steady force, and partially non-dissipative like the added-mass force and used to modify the viscous microscale flow in the vicinity of the particle. (Note that the kinetic energy transfer due to the added-mass force is used to modify the inviscid microscale flow around the particle.) The former portion is irreversible and positively contributes to the fluid internal energy; while the latter portion is reversible and contributes to kinetic energy exchange between the macroscale and microscale, see Fig. 1. Due to the complex nature of the viscous-unsteady force, it is difficult to separate the two contributions in the time domain (see Parmar et al. ${ }^{37}$ ). Over long time scale, the dissipation portion can be expected to dominate and the following closure is appropriate for $g_{v u}$ as

$$
g_{v u}=\mathbf{f}_{v u} \cdot \mathbf{u}_{p} .
$$

Based on the above discussion of the energy implications of the inter-phase coupling forces, finally we obtain the following expression for $G_{f p, i}$ to be used in (3) in the context of PPA:

$$
G_{f p, i}=\mathbf{F}_{s g, i} \cdot \mathbf{u}_{p, i}+\mathbf{F}_{q s, i} \cdot \mathbf{u}_{p, i}+\mathbf{F}_{a m, i} \cdot \mathbf{u}_{f}+\mathbf{F}_{v u, i} \cdot \mathbf{u}_{p, i} \cdot
$$

Similarly for the two-fluid approach, the total and internal energy equations, Eqs. (18) and (28), can be rewritten as

$$
\begin{gathered}
\frac{\partial\left(\rho_{f} \phi_{f} E_{f}\right)}{\partial t}+\nabla \cdot\left(\rho_{f} \phi_{f} \mathbf{u}_{f} E_{f}\right)-\nabla \cdot\left(\phi_{f} \boldsymbol{\sigma}_{f} \cdot \mathbf{u}_{f}\right)-\phi_{f} \nabla \cdot \mathbf{q}_{f}= \\
\boldsymbol{\sigma}_{f}: \nabla\left(\phi_{p} \mathbf{u}_{p}\right)-\rho_{p} \phi_{p}\left[\left(\mathbf{f}_{q s}+\mathbf{f}_{v u}\right) \cdot \mathbf{u}_{p}+\mathbf{f}_{a m} \cdot \mathbf{u}_{f}\right]-\rho_{p} \phi_{p}\left(q_{q s}+q_{d u}\right)
\end{gathered}
$$

and

$$
\rho_{f} \phi_{f} \frac{D e_{f}}{D t}=\phi_{f} \nabla \cdot \mathbf{q}_{f}+\sigma_{f}: \nabla \mathbf{u}_{m}+\rho_{p} \phi_{p}\left(\mathbf{f}_{q s}+\mathbf{f}_{v u}\right) \cdot\left(\mathbf{u}_{f}-\mathbf{u}_{p}\right)-\rho_{p} \phi_{p}\left(q_{q s}+q_{d u}\right) .
$$

It must be stressed that only the sum of (a) the energy of the particles, (b) the macroscale energy of the fluid, and (c) the energy of the microscale motion of the fluid is conserved. In other words, only the sum of Eqs. (21), (35), and $\mathbf{f}_{a m} \cdot\left(\mathbf{u}_{p}-\mathbf{u}_{f}\right)$ add to zero, where the last two correspond to the macroscale and microscale total energy of the fluid flow.

\section{CHARACTERISTIC SCALES OF TURBULENT DISPERSED MULTIPHASE FLOWS}

\section{A. Scales of particle motion and thermal evolution}

The particle mechanical time scale, $\tau_{p m}$, is generally defined as the time it takes for a particle, instantaneously released in a steady uniform stream, to accelerate and the relative velocity to decay by factor $(1 / e)$. Under such simplified condition, Eqs. (7)-(11), in the absence of viscous unsteady force, can be solved to define the time scale for particle motion as

$$
\tau_{p m}=\frac{d_{p}^{2}}{12 \beta v_{f}} \frac{1}{\Phi\left(\operatorname{Re}_{p}\right)},
$$

where $\beta=3 /\left(2 \rho_{p} / \rho_{f}+1\right)$. For a heavy particle $\beta=0$, for neutrally buoyant particles $\beta=1$, and for bubbles, $\beta=3$.

Similarly, the particle thermal time scale can be defined as the time it takes for a particle with initial temperature $T_{p, 0}$, instantaneously released in a steady fluid flow of uniform temperature $T_{f}$, to be heated or cooled and the relative temperature reaches $(1 / e)$ times the original temperature difference. Eqs. (12)-(15), in the absence of diffusive-unsteady contribution, can be solved and the thermal time scale of the particle can be written as

$$
\tau_{p \theta}=\frac{d_{p}^{2}}{12 \beta_{\theta} \alpha_{f}} \frac{1}{\Psi\left(\operatorname{Re}_{p}, \operatorname{Pr}_{f}\right)},
$$

where $\beta_{\theta}=\rho_{f} C_{f} /\left(\rho_{p} C_{p}\right)$ is the volumetric heat capacity ratio between the fluid and particles. The thermal diffusivity of the fluid is given by $\alpha_{f}=k_{f} /\left(\rho_{f} C_{f}\right)$. 


\section{B. Scales of turbulent motion and thermal evolution}

In turbulent flows, there exists eddies of size varying from the Kolmogorov microscale to the integral scale. The Kolmogorov microscale is expressed as $\eta=\left(v_{f} / \epsilon\right)^{1 / 4}$, which depends on the kinematic viscosity $v_{f}$ and the dissipation rate $\epsilon$. At large Reynolds number, an inertial subrange exists between the Kolmogorov and the integral scales, where the effect of viscosity on the energy spectral density can be neglected.

A similar subrange also exists in the spectral density of the variance of temperature fluctuations, (i.e., in the spectra of $k_{\theta}=\overline{T_{f}^{\prime 2}}$ ), which is the thermal analog of the turbulent kinetic energy. In this inertial-convective subrange, the temperature fluctuation is dictated by the turbulent eddies, and the influence of viscosity and thermal diffusivity on $k_{\theta}$ can be ignored. ${ }^{18,19}$ A thermal microscale similar to the Kolmogorov microscale can then be defined as $\eta_{\theta}=\left(\alpha_{f} / \epsilon\right)^{1 / 4}$. In a fluid of $\operatorname{Pr}_{f}<1$, $\eta<\eta_{\theta}$ and the temperature fluctuations will be diffused before the cascade reaches the smallest eddies which then are dissipated. In case of $\operatorname{Pr}_{f}>1, \eta>\eta_{\theta}$ and an additional inertial-diffusive subrange will appear, in which the spectral density of $k_{\theta}$ depends on $\alpha_{f}$ but not $v_{f}{ }^{38}$ Due to the focus of the present paper on inter-phase energy coupling, here we consider the simple case of $\operatorname{Pr}_{f}=1$ (and $\eta=\eta_{\theta}$ ). The scaling approach to be presented can be extended to the more general case of $\operatorname{Pr}_{f} \neq 1$.

Based on the hypothesis that the dissipation rates of $k$ and $k_{\theta}$ are constant within the inertialconvective subrange, the velocity and temperature scales of an eddy of size $l(\eta<l<L$, where $L$ is the integral length scale) can be expressed as

$$
\begin{aligned}
& u_{l}=\left(\epsilon \tau_{l}\right)^{1 / 2}, \\
& T_{l}=\left(\epsilon_{\theta} \tau_{l}\right)^{1 / 2},
\end{aligned}
$$

where $\epsilon_{\theta}$ is the dissipation of $k_{\theta}$, and $\tau_{l}$ is the time scale for the $l$-size eddy. It can be shown that $u_{L} / u_{\eta}=T_{L} / T_{\eta}=\left(\tau_{L} / \tau_{\eta}\right)^{1 / 2}$. Since $u_{l}=l / \tau_{l}$, then Eqs. (39) and (40) can be rewritten as

$$
\begin{aligned}
& u_{l}=l^{1 / 3} \epsilon^{1 / 3}, \\
& T_{l}=l^{1 / 3} \epsilon_{\theta}^{1 / 2} \epsilon^{-1 / 6} .
\end{aligned}
$$

The scales of mechanical and thermal acceleration for $l$-size eddy are then estimated as $(D u / D t)_{l}=$ $u_{l} / \tau_{l}$ and $(D T / D t)_{l}=T_{l} / \tau_{l}$.

\section{Estimates of relative temperature and thermal acceleration}

In a turbulent multiphase flow, a particle will interact with eddies of different sizes. In order to estimate the relative velocity and relative acceleration between the particle and fluid, Ling et al. ${ }^{14}$ advanced a hypothesis that a particle will mechanically respond to an eddy of size $l$ only if the mechanical Stokes number corresponding to the $l$-size eddy $\left(\mathrm{St}_{m, l}=\tau_{m} / \tau_{l}\right)$ is less than unity. In fact, this hypothesis has also been used in other previous works and proved to be effective. ${ }^{39-42} \mathrm{~A}$ similar hypothesis is made here to estimate the scales of relative temperature $\left|T_{f}-T_{p}\right|$ and relative thermal acceleration $\left|D T_{f} / D t-d T_{p} / d t\right|$. It is considered that only if the thermal Stokes number $\left(\mathrm{St}_{\theta, l}=\tau_{p \theta} / \tau_{l}\right)$ is less than unity, the particle will thermally respond to the temperature fluctuation induced by the $l$-size eddy. In such a case, the relative temperature and thermal acceleration can be estimated from the Equilibrium Eulerian Approximation (EEA) as (see the work of Ferry and Balachandar ${ }^{43}$ for thermal equilibrium approximation)

$$
T_{f}-T_{p}=\tau_{p \theta}\left(1-\beta_{\theta}\right) \frac{D T_{f}}{D t}+\mathrm{O}\left(S t_{m} S t_{\theta}, S t_{\theta}^{2}\right) .
$$

The above equation is an asymptotic solution of Eq. (12) with $\mathrm{St}_{m}$ and $\mathrm{St}_{\theta}$ as small parameters. A simple interpretation of Eq. (43) is that when the particle temperature is in equilibrium with the fluid temperature, their temperature difference is dictated by particle's inability to respond to the local fluid temperature variation. Validation tests and range of applicability of EEA for turbulent flows can be found in the work of Ferry and Balachandar. ${ }^{43}$ Furthermore, it can be easily shown that EEA is equivalent to the assumption $D T_{f} / D t=d T_{p} / d t$. 
In contrast, when $\mathrm{St}_{\theta, l}>1$, the particle is considered thermally too sluggish to respond to the temperature variation induced by the $l$-size eddy. Then the relative temperature will be dictated by the temperature scale of the $l$-size eddy. Similarly, the thermal acceleration of the particle in response to the $l$-size eddy is negligible, and the relative thermal acceleration is dictated by the fluid thermal acceleration alone.

In summary, the relative temperature and the thermal acceleration between the fluid and particle for $\mathrm{St}_{\theta, l}<1$ and $\mathrm{St}_{\theta, l}>1$ can be approximated as

$$
\begin{gathered}
\left\{\begin{array}{l}
\left|T_{f}-T_{p}\right|_{l} \approx \tau_{p \theta}\left|1-\beta_{\theta}\right| \frac{T_{l}}{\tau_{l}}, \\
\left|\frac{D T_{f}}{D t}-\frac{d T_{p}}{d t}\right|_{l} \approx 0,
\end{array}\right\} \quad \text { if } \mathrm{St}_{\theta, l}<1, \\
\left\{\begin{array}{l}
\left|T_{f}-T_{p}\right|_{l} \approx\left|1-\beta_{\theta}\right| T_{l}, \\
\left|\frac{D T_{f}}{D t}-\frac{d T_{p}}{d t}\right|_{l} \approx \frac{T_{l}}{\tau_{l}},
\end{array}\right\} \quad \text { if } \mathrm{St}_{\theta, l}>1 .
\end{gathered}
$$

\section{Different regimes in turbulent multiphase flows}

With the above results of relative temperature and thermal acceleration in response to the $l$-size eddy, we can obtain estimates of the maximum relative temperature and thermal acceleration for a particle subjected to a range of turbulent eddies. Similar analysis has been performed by Ling et al. ${ }^{14}$ to estimate the maximum relative velocity and particle Reynolds number. Based on the values of mechanical and thermal time scales of the particle $\left(\tau_{p m}\right.$ and $\left.\tau_{p \theta}\right)$ in relation to the typical turbulence time scales $\left(\tau_{\eta}\right.$ and $\left.\tau_{L}\right)$, we can define three different regimes of particle mechanical and thermal behavior. Since the mechanical behavior has been discussed by Ling et al. ${ }^{14}$ here we will focus only on the thermal behavior in the three regimes.

- Regime I particles $\left(\left\{\tau_{p \theta}, \tau_{p m}\right\}<\tau_{\eta}\right)$ : In this regime, $\mathrm{St}_{\theta, L}<\mathrm{St}_{\theta, \eta}<1$, where $\mathrm{St}_{\theta, L}=\tau_{p \theta} / \tau_{L}$ and $\mathrm{St}_{\theta, \eta}=\tau_{p \theta} / \tau_{\eta}$. Since $\tau_{\eta}$ is the smallest time scale in the fluid flow, the particle will respond to the temperature fluctuation of every eddy in the flow. Therefore, the relative temperature and the relative thermal acceleration corresponding to every eddy can be estimated by Eq. (44) and we have

$$
\begin{aligned}
\frac{\left|T_{f}-T_{p}\right|_{l}}{T_{\eta}} & \approx\left|1-\beta_{\theta}\right| \mathrm{St}_{\theta, \eta}^{1 / 2}\left(\frac{\tau_{l}}{\tau_{p \theta}}\right)^{-1 / 2}, \\
\left|\frac{D T_{f}}{D t}-\frac{d T_{p}}{d t}\right|_{l} & \approx 0,
\end{aligned}
$$

which are shown in Fig. 2. Since the relative thermal acceleration in this regime is identical to zero, it is not shown in the log-log plot of Fig. 2(b). The maximum relative temperature in this regime, thus, corresponds to the Kolmogorov time scale, and can be given as

$$
\begin{gathered}
\left|T_{f}-T_{p}\right|_{\max , \mathrm{I}} \approx \tau_{p \theta}\left|1-\beta_{\theta}\right| \frac{T_{\eta}}{\tau_{\eta}}, \\
\left|\mathbf{u}_{f}-\mathbf{u}_{p}\right|_{\max , \mathrm{I}} \approx \tau_{p m}|1-\beta| \frac{u_{\eta}}{\tau_{\eta}}, \\
\operatorname{Re}_{p, \mathrm{I}} \Phi\left(\operatorname{Re}_{p, \mathrm{I}}\right) \approx \frac{|1-\beta|}{12 \beta}\left(\frac{d_{p}}{\eta}\right)^{3} .
\end{gathered}
$$

For completeness the scaling of the maximum relative velocity and the particle Reynolds number have also been presented from Ling et al. ${ }^{14}$

- Regime III particles $\left(\left\{\tau_{p \theta}, \tau_{p m}\right\}>\tau_{L}\right)$ : In this regime, $1<\mathrm{St}_{\theta, L}<\mathrm{St}_{\theta, \eta}$. The particle thermal time scale exceeds all turbulence time scales and thus the particle does not thermally respond to any eddy. The relative temperature is then determined by the temperature fluctuation of the eddy as given in Eq. (45). In this regime, we have 

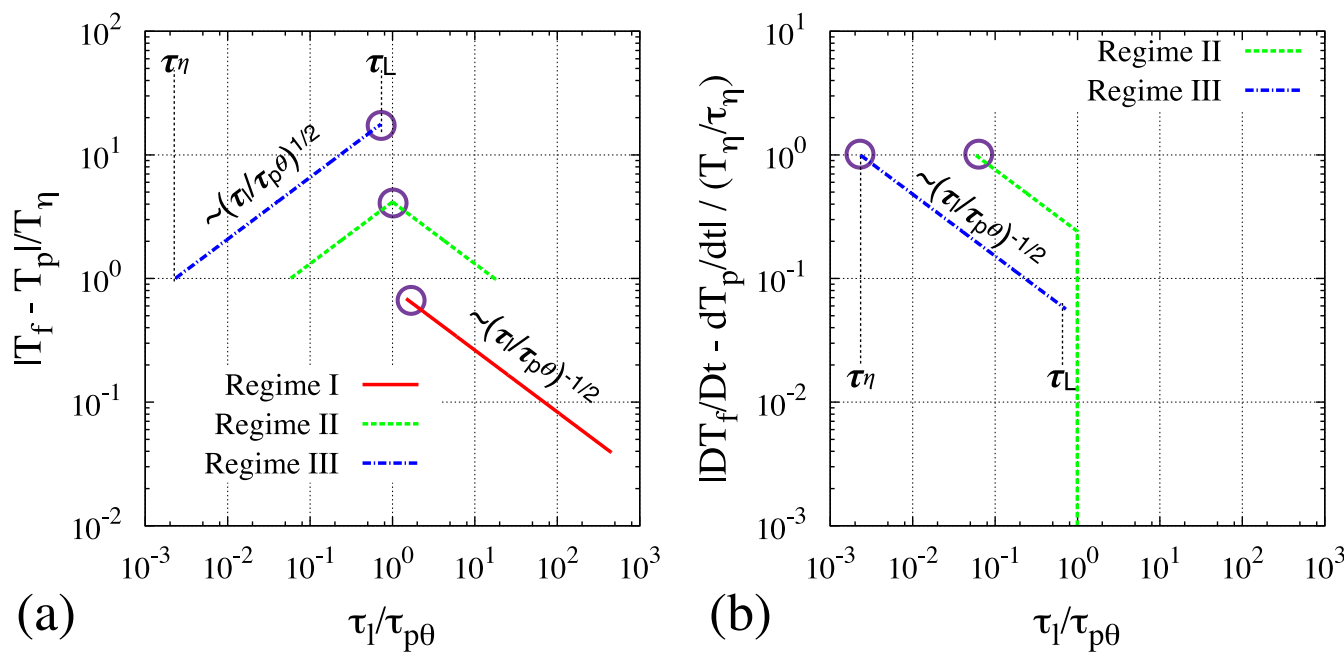

FIG. 2. Estimates of relative (a) temperature and (b) thermal acceleration between fluid and particles as functions of $\tau_{l} / \tau_{p \theta}$ for the three different regimes (regime I: $\left(\tau_{p \theta}<\tau_{\eta}\right)$; regime II: $\tau_{\eta}<\tau_{p \theta}<\tau_{L}$; regime III: $\left.\tau_{p \theta}>\tau_{L}\right)$. The maximum values in each regime are marked with a open circle. Note that the relative thermal acceleration of regime I particles is identical to zero and thus is not shown in the log-log plot. The results plotted in this example are for $\tau_{\eta}=1 \mathrm{~ms}, \tau_{L}=316 \mathrm{~ms}$, and $\tau_{p \theta}=0.692,17.3,433 \mathrm{~ms}$ for regimes I, II, and III, respectively. These parameters are corresponding to sand particles $\left(d_{p}=10,50,250 \mu \mathrm{m}\right.$ for regimes I, II, and III) in air for $\operatorname{Re}_{L}=10^{5}$.

$$
\begin{gathered}
\frac{\left|T_{f}-T_{p}\right|_{l}}{T_{\eta}} \approx\left|1-\beta_{\theta}\right| \mathrm{st}_{\theta, \eta}^{1 / 2}\left(\frac{\tau_{l}}{\tau_{p \theta}}\right)^{1 / 2}, \\
\left|\frac{D T_{f}}{D t}-\frac{d T_{p}}{d t}\right|_{l} \frac{\tau_{\eta}}{T_{\eta}} \approx \mathrm{st}_{\theta, \eta}^{-1 / 2}\left(\frac{\tau_{l}}{\tau_{p \theta}}\right)^{-1 / 2},
\end{gathered}
$$

which are also shown in Fig. 2. Since the largest fluid temperature fluctuation is $T_{L}$, see Eq. (42), the maximum relative temperature in this regime can be approximated as

$$
\begin{aligned}
\left|T_{f}-T_{p}\right|_{\max , \text { III }} & \approx\left|1-\beta_{\theta}\right| T_{L}, \\
\left|\mathbf{u}_{f}-\mathbf{u}_{p}\right|_{\max , \mathrm{III}} & \approx|1-\beta| u_{L}, \\
\operatorname{Re}_{p, \text { III }} & \approx|1-\beta|\left(\frac{d}{\eta}\right)\left(\frac{L}{\eta}\right)^{1 / 3} .
\end{aligned}
$$

- Regime II particles $\left(\tau_{\eta}<\left\{\tau_{p \theta}, \tau_{p m}\right\}<\tau_{L}\right)$ : In this regime, $\mathrm{St}_{\theta, L}<1<\mathrm{St}_{\theta, \eta}$. The particle responds to the temperature fluctuation induced by the large eddies in the flow but do not respond to the small ones. There exists an eddy of size $l^{*}\left(\eta<l^{*}<L\right)$, whose time scale matches the particle thermal time scale, i.e., $\tau_{l^{*}}=\tau_{p \theta}$. The particle will respond to the larger eddies with $\tau_{l}>\tau_{l^{*}}$, and the relative temperature and thermal acceleration are represented by Eqs. (46) and (47). On the other hand, the particle does not respond to the smaller eddies of $\tau_{l}<\tau_{l^{*}}$, and the relative temperature and thermal acceleration are as given by Eqs. (51) and (52). It can be shown that the relative temperature increases with $\tau_{l}$ for $\tau_{l}<\tau_{l^{*}}$ and decreases for $\tau_{l}>\tau_{l^{*}}$ (see Fig. 2(a)). Then the maximum relative temperature is reached at $\tau_{l^{*}}$ and the value of which is given as

$$
\begin{aligned}
\left|T_{f}-T_{p}\right|_{\text {max }, \mathrm{II}} & \approx \tau_{p \theta}\left|1-\beta_{\theta}\right| \frac{T_{l^{*}}}{\tau_{l^{*}}}=\left|1-\beta_{\theta}\right| T_{l^{*}}, \\
\left|\mathbf{u}_{f}-\mathbf{u}_{p}\right|_{\text {max }, \mathrm{II}} & \approx \tau_{p m}|1-\beta| \frac{u_{l^{*}}}{\tau_{l^{*}}}=|1-\beta| u_{l^{*}} . \\
\operatorname{Re}_{p, \mathrm{II}} \sqrt{\Phi\left(\operatorname{Re}_{p, \mathrm{II}}\right)} & \approx \frac{|1-\beta|}{\sqrt{12 \beta}}\left(\frac{d_{p}}{\eta}\right)^{2} .
\end{aligned}
$$

The above three major regimes are defined based on the assumption that $\tau_{p m}$ and $\tau_{p \theta}$ are close, so that they are either both smaller than $\tau_{\eta}\left(\tau_{L}\right)$ or both larger than $\tau_{\eta}\left(\tau_{L}\right)$. This assumption is valid 
for a wide range of particle materials. For example, at low Reynolds numbers, $\tau_{p m} / \tau_{p \theta}$ for glass and copper particles, immersed in cool air, are 1.13 and 2.44, respectively. Nevertheless, there exist cases where the particle mechanical and thermal time scales are very different. In such cases, minor regimes such as $\tau_{p m}<\tau_{\eta}$ and $\tau_{\eta}<\tau_{p \theta}<\tau_{L}$ exist. In the present paper, we will focus only on the three important major regimes. Similar analysis can be easily extended to the minor regimes if they are of interest.

\section{INTEGRAL REPRESENTATION OF DIFFUSIVE-UNSTEADY HEAT TRANSFER}

In this section, different representations of the diffusive-unsteady heat transfer are discussed. The conventional formulation of $Q_{d u}$, as shown in Eq. (15), involves a convolutional integral and thus is computationally costly. It is therefore of great interest to see if it is possible to simplify the calculation of $Q_{d u}$ and under what conditions such a simplification can be justified.

For finite but small particle Peclet number, the diffusive-unsteady kernel $K_{d u}$ appearing in Eq. (15) is given by Feng and Michaelides, ${ }^{11}$

$$
K_{d u}\left(t / \tau_{d u}\right)=\frac{\exp \left(-t / \tau_{d u}\right)}{\sqrt{\pi\left(t / \tau_{d u}\right)}}-\operatorname{erfc}\left(\sqrt{t / \tau_{d u}}\right),
$$

where the diffusive-unsteady time scale is defined as

$$
\tau_{d u}=\frac{d_{p}^{2}}{\alpha_{f}} \frac{4}{\mathrm{Pe}_{p}^{2}} .
$$

The diffusive-unsteady kernel given in Eq. (59) is shown in Fig. 3. It is seen that at short time $\left(t \ll \tau_{d u}\right), K_{d u}$ reduces to the form in the Stokes limit

$$
\lim _{t / \tau_{d u} \rightarrow 0} K_{d u}\left(t / \tau_{d u}\right)=\frac{1}{\sqrt{\pi\left(t / \tau_{d u}\right)}}
$$

and $K_{d u}$ decays as $t^{-1 / 2}$.

At long time $t \gg \tau_{d u}$, both the terms on the right hand side of Eq. (59) approach zero exponentially. Furthermore, it can be shown that the first term becomes identical to the second with a opposite sign as $t / \tau_{d u} \rightarrow \infty$. Thus the sum of them further accelerates the decay of $K_{d u}$.

Therefore, $\tau_{d u}$ can be viewed as the time scale at which $K_{d u}$ switches from the slow $t^{-1 / 2}$ decay to the fast exponential decay. If the particle and fluid thermal acceleration time scales are much larger than $\tau_{d u}$, then the relative thermal acceleration can be taken out of the convolution integral and Eq. (15) can be reduced to the following simpler form:

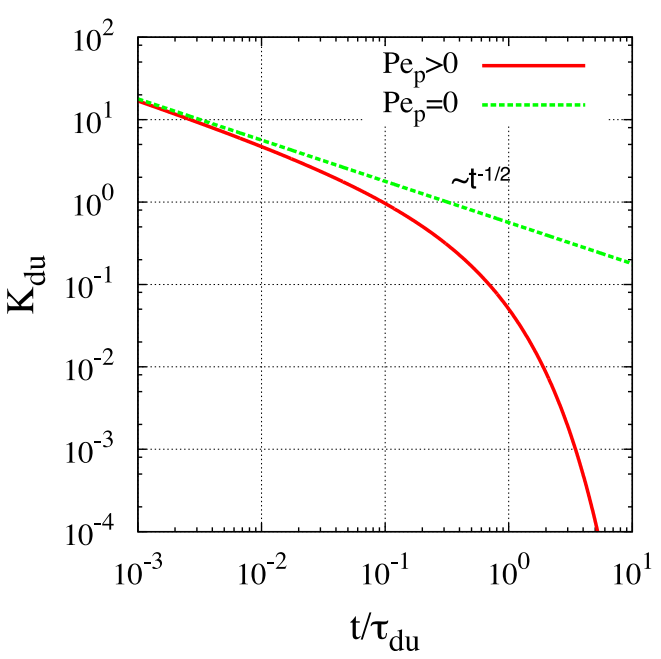

FIG. 3. Diffusive-unsteady kernel as a function of $t / \tau_{d u}$. 


$$
Q_{d u}=V_{p} \rho_{f} C_{f} C_{d u}\left(\frac{D T_{f}}{D t}-\frac{d T_{p}}{d t}\right) .
$$

A similar simplified formulation for diffusive-unsteady heat transfer has been proposed by Balachandar and Ha. ${ }^{12}$ Here the effective diffusive-unsteady coefficient, $C_{d u}$, is defined as

$$
C_{d u}=\frac{24}{\mathrm{Pe}_{p}} \int_{0}^{\infty} K_{d u}\left(\xi / \tau_{d u}\right) d\left(\xi / \tau_{d u}\right)=\frac{12}{\mathrm{Pe}_{p}} .
$$

Note that $C_{d u} \rightarrow \infty$ when $\mathrm{Pe}_{p}=0$. Since $\tau_{d u} \rightarrow \infty$ in the limit of $\mathrm{Pe}_{p}=0$, the kernel function always decays as $t^{-1 / 2}$ and as a result the integral of the kernel diverges. Therefore, finite $C_{d u}$ only exists for finite $\mathrm{Pe}_{p}$.

It can be argued that the Kolmogorov time scale $\left(\tau_{\eta}\right)$ is the smallest relevant characteristic time scale of relative thermal acceleration. In the case $\tau_{p \theta}>\tau_{\eta}$, particle response is slow and the time scale of relative thermal acceleration is dictated by that of the fluid. Even in the case of fast particle response time (i.e., $\tau_{p \theta}<\tau_{\eta}$ ), since thermal fluctuations in the particle are induced only by the thermal fluctuations of the fluid, the characteristic time scales of thermal acceleration of both the fluid and the particle are $\tau_{\eta}$. As a result, if $\tau_{d u} \ll \tau_{\eta}$, then the diffusive-unsteady heat transfer can be accurately approximated by the simpler expression given in Eq. (62). Otherwise, the conventional and more complex integral form, Eq. (15), must be used. Therefore, the ratio $\tau_{\eta} / \tau_{d u}$ plays a critical role in determining whether the simplified equation can be used or not. In the present study, we consider the simple case that $\operatorname{Pr}_{f}=1$, so that $\tau_{\eta}=\eta_{/} v_{f}=\eta / \alpha_{f}$, then it is shown that

$$
\frac{\tau_{\eta}}{\tau_{d u}}=\frac{1}{4}\left(\frac{\eta}{d_{p}}\right)^{2} \operatorname{Re}_{p}^{2} .
$$

It is seen that $\operatorname{Re}_{p}$ is required to estimate $\tau_{\eta} / \tau_{d u}$. With the approximations of $\operatorname{Re}_{p}$ given in Eqs. (50), (58), and (55), $\tau_{\eta} / \tau_{d u}$ can be estimated in the three regimes as

- Regime I

$$
\frac{\tau_{\eta}}{\tau_{d u}} \approx \frac{|1-\beta|^{2}}{576 \beta^{2} \Phi^{2}}\left(\frac{d_{p}}{\eta}\right)^{4},
$$

- Regime II

$$
\frac{\tau_{\eta}}{\tau_{d u}} \approx \frac{|1-\beta|^{2}}{48 \beta \Phi}\left(\frac{d_{p}}{\eta}\right)^{2},
$$

- Regime III

$$
\frac{\tau_{\eta}}{\tau_{d u}} \approx \frac{|1-\beta|^{2}}{4}\left(\frac{L}{\eta}\right)^{2 / 3} .
$$

Therefore, the time scale ratio $\tau_{\eta} / \tau_{d u}$ is a function of $d_{p} / \eta, \rho_{p} / \rho_{f}$, and $L / \eta$, which is plotted in Fig. 4(a). The finite Reynolds number correction is taken to be $\Phi=1$ in Fig. 4.

Several important observations can be made. First of all, a horizontal line can be drawn for a chosen threshold $\left(\tau_{\eta} / \tau_{d u}\right)_{t h}$ (for example, see the line $\left(\tau_{\eta} / \tau_{d u}\right)=1$ in Fig. 4(a)). For cases falling above the line, the simpler form of $Q_{d u}$, Eq. (62) can be used. It is observed that $\tau_{\eta} / \tau_{d u}$ is, in general, not very sensitive to $L / \eta$, except when the particle size is large compared to the Kolmogorov length scale, falling in regime III.

Furthermore, $\tau_{\eta} / \tau_{d u}$ increases monotonically with $d_{p} / \eta$. As a result, there exists a critical diameter, $d_{p, d u}^{*}$, and for particles larger than $d_{p, d u}^{*}$ the diffusive-unsteady heat transfer can be calculated without the convolution integral. If we set the threshold here as $\left(\tau_{\eta} / \tau_{d u}\right)=1$, then it can be shown from Eqs. (65)-(67) that $d_{p, d u}^{*}$ corresponds to regime II. If the effect of $\Phi$ is further neglected, we have

$$
d_{p, d u}^{*} / \eta=\frac{\sqrt{48 \beta}}{|1-\beta|} .
$$

It can be shown that the particle time scale corresponding to this critical diameter satisfies $\tau_{p m}^{*} / \tau_{\eta}=$ $4 /|1-\beta|^{2} \geq 1$ for all $\beta$ and thus validates the assertion that $d_{p, d u}^{*}$ is in regime II. As shown in 

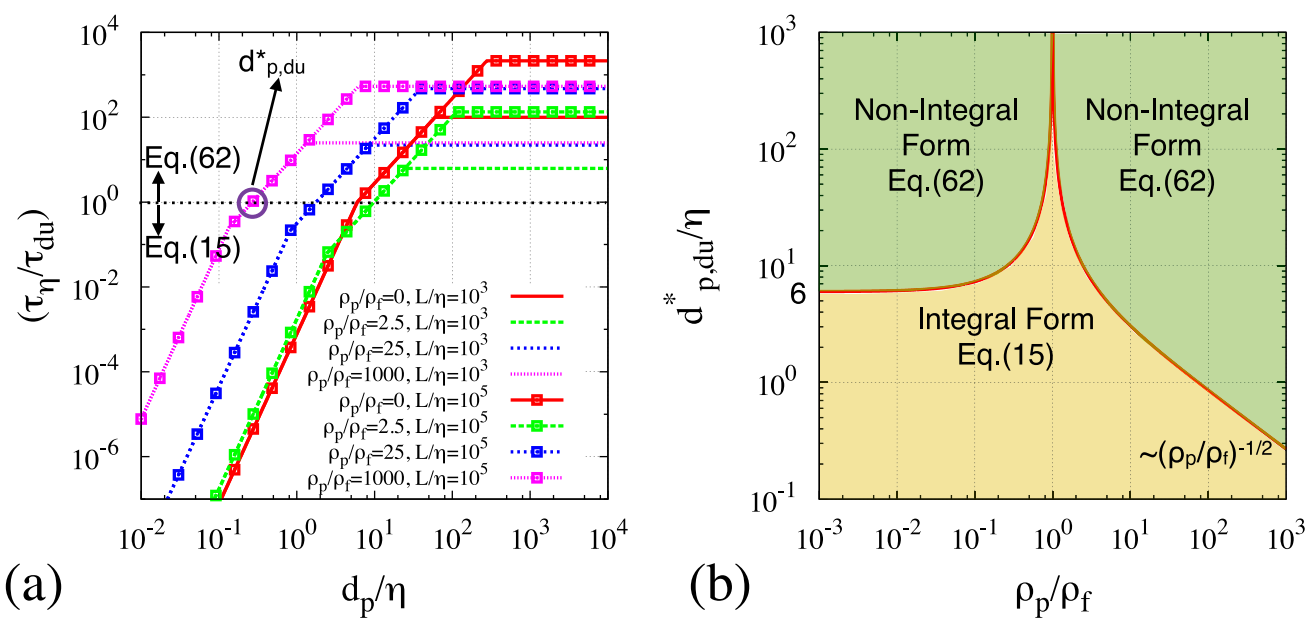

FIG. 4. (a) The time scale ratio $\tau_{\eta} / \tau_{d u}$ as a function of $d_{p} / \eta, \rho_{p} / \rho_{f}$, and $L / \eta$ and (b) the critical particle diameter $d_{p, d u}^{*}$ as a function of $\rho_{p} / \rho_{f}$ when the threshold is set to be $\left(\tau_{\eta} / \tau_{d u}\right)=1$.

Eq. (68), $d_{p, d u}^{*}$ depends only on $\rho_{p} / \rho_{f}$ (or $\beta$ ). The variation of $d_{p, d u}^{*}$ as a function of $\rho_{p} / \rho_{f}$ is plotted in Fig. 4(b). For particle sizes below and above $d_{p, d u}^{*}$ we can use the integral (Eq. (15)) and non-integral (Eq. (62)) forms of diffusive-unsteady heat transfer, respectively.

In general, the integral form is required for very small particles and the non-integral form can be used for very large particles (except for the special case that $\rho_{p} / \rho_{f}=1$ ). For bubbly flows $\left(\rho_{p} / \rho_{f} \ll 1\right), d_{p, d u}^{*} / \eta \rightarrow 6$, therefore, the integral form, Eq. (15), is generally needed unless the bubble size is six times or more larger than the Kolmogorov length scale. For gas-particle flows $\left(\rho_{p} / \rho_{f} \gg 1\right)$, since $d_{p, d u}^{*} / \eta$ decrease as $\left(\rho_{p} / \rho_{f}\right)^{-1 / 2}$, there exist a wide range of particle size where the simpler form Eq. (62) can be used. For example, when $\rho_{p} / \rho_{f}=10^{3}$, the non-integral form of diffusive-unsteady heat transfer can be used even for particles smaller than the Kolmogorov length scale. For neutrally buoyant particles $\left(\rho_{p} / \rho_{f}=1\right)$, since the relative velocity is identical to zero, both $\tau_{d u}$ and $d_{p, d u}^{*} / \eta$ approach infinity.

In general, PPA is theoretically well grounded only in the limit when the particle size is much smaller than the grid resolution. Extension of PPA to $d / \eta>1$ requires additional modeling efforts, such as including the stochastic contribution to the particle motion, ${ }^{44}$ which is out of the scope of the present paper. For cases $d / \eta>1$, the diffusive-unsteady heat transfer formulations (Eqs. (15) and (62)) given here can at most serve as the deterministic portion of the overall diffusive-unsteady contribution. Therefore it is still interesting to show under what condition the convolution integral formulation can be simplified.

\section{SCALING ANALYSIS OF INTER-PHASE ENERGY COUPLING}

Strictly speaking, due to the inter-phase momentum and energy transfer, the fluid and the particles are always two-way coupled. On one hand, the motion and thermal evolution of the particles are driven by the fluid flow (forward coupling); on the other hand, the particles influence the fluid velocity and temperature evolution (backward coupling). The back effects of the particles on the fluid are represented by the source terms $-\sum_{i}^{N_{p}} \mathbf{F}_{f p, i} / \mathbb{V}$ and $-\frac{1}{\mathbb{V}} \sum_{i}^{N_{p}}\left(G_{f p, i}+Q_{f p, i}\right)$ on the right hand sides of Eqs. (2) and (3) (or those of Eqs. (17) and (18)). If the back effects are not important, then the particles and fluid can be approximated as one-way coupled and the above source terms can be ignored (as compared to the remaining terms). Nevertheless, such simplifications must be made with great caution, otherwise neglecting these contributions may lead to significant errors. In this section, scaling estimates are established to access the importance of different contributions of heat transfer in forward and backward energy coupling, by which one can determine whether unsteady heat transfer to particles and its back effect on the fluid can be ignored. Similar analysis for the importance of unsteady forces and their backward coupling to the fluid momentum balance has been considered by Ling et al. ${ }^{14}$ 


\section{A. Importance of unsteady heat transfer in forward energy coupling}

The importance of the unsteady contributions to heat transfer in forward energy coupling is evaluated in terms of the ratio between the unsteady and quasi-steady contributions.

\section{Undisturbed-unsteady heat transfer}

From (13) to (15), and with the definition of thermal time scale of the particle given in (38), the ratio between the undisturbed-unsteady to the quasi-steady contribution can be expressed as

$$
\frac{\left|Q_{u u}\right|}{\left|Q_{q s}\right|}=\beta_{\theta} I_{u u}
$$

where $I_{u \iota}$ is a correction function, defined as

$$
I_{u u}=\frac{\tau_{p \theta}\left|D T_{f} / D t\right|}{\left|T_{f}-T_{p}\right|} .
$$

It is seen that the conventional estimate that the fluid-to-particle volumetric heat capacity ratio (i.e., $\beta_{\theta}$ ) is sufficient to determine the importance of the unsteady heat transfer is valid only if the correction factor $I_{u u} \sim O(1)$. With Eqs. (44) and (45), we obtain

$$
I_{u u}= \begin{cases}\frac{\mathrm{St}_{\theta, l}}{\left|1-\beta_{\theta}\right|}, & \text { if } \tau_{l}<\tau_{p \theta}, \\ \frac{1}{\left|1-\beta_{\theta}\right|}, & \text { if } \tau_{l}>\tau_{p \theta} .\end{cases}
$$

It is seen that the denominator $\left|1-\beta_{\theta}\right|$ approaches 1 for small $\beta_{\theta}$ (such as for the case of gasparticle flows) and to $\beta_{\theta}$ for large $\beta_{\theta}$ (such as for the case of bubbly flows). For the special case of $\beta_{\theta}=1$, namely, $\rho_{p} C_{p}=\rho_{f} C_{f}$, the quasi-steady contribution to heat transfer is identically zero and thus $I_{u u} \rightarrow \infty$.

For regime I particles, since $\tau_{p \theta}$ is smaller than all turbulent time scales, $I_{u u}=1 /\left|1-\beta_{\theta}\right|$, while for regime III particles, since $\tau_{p \theta}$ is larger than all turbulent time scales, $I_{u u}=\mathrm{St}_{\theta, l} /\left|1-\beta_{\theta}\right|$. For particles in regime II, $I_{u u}=1 /\left|1-\beta_{\theta}\right|$ for the eddies with $\tau_{l}$ larger than $\tau_{p \theta}$ and $I_{u u}=\mathrm{St}_{\theta, l} /\left|1-\beta_{\theta}\right|$ for the eddies with $\tau_{l}$ smaller than $\tau_{p \theta}$. The behavior of the correction function is shown in Fig. 5(a). The largest value of the correction function always corresponds to the smallest turbulent eddy, i.e., the Kolmogorov scale eddy, and the maximum value of $I_{u u}$ for all the three regimes can be
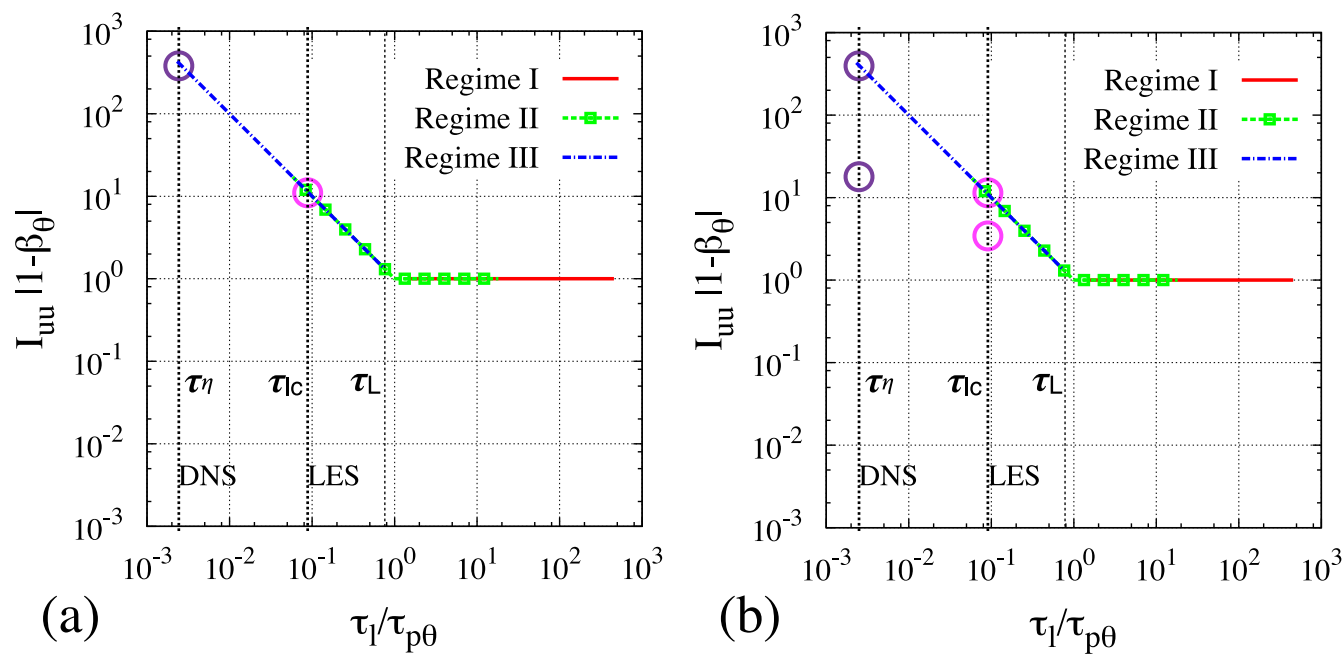

FIG. 5. Correction functions for (a) undisturbed-unsteady heat transfer $\left(I_{u u}\right)$ and (b) diffusive-unsteady heat transfer $\left(I_{d u, n i n t}\right.$ and $I_{d u \text {,int }}$ ), as functions of $\tau_{l} / \tau_{p m}$ in three regimes. The results plotted in this example are for $\tau_{\eta}=1 \mathrm{~ms}, \tau_{L}=316 \mathrm{~ms}$, and $\tau_{p \theta}=0.692,17.3,433 \mathrm{~ms}$ for regimes I, II, and III, respectively. These parameters are corresponding to sand particles $\left(d_{p}=10,50,250 \mu \mathrm{m}\right.$ for regimes I, II, and III) in air for $\operatorname{Re}_{L}=10^{5}$. 
summarized as

$$
I_{u u, \max }=\frac{\max \left(1, \mathrm{St}_{\theta, \eta}\right)}{\left|1-\beta_{\theta}\right|}
$$

\section{Diffusive-unsteady heat transfer}

Similarly, the importance of the diffusive-unsteady heat transfer is evaluated through the ratio $Q_{d u} / Q_{q s}$. As explained in Section IV, the diffusive-unsteady heat transfer can be expressed in the non-integral form (Eq. (62)) or the integral (Eq. (15)) form depending on the time scale ratio $\tau_{\eta} / \tau_{d u}$. The latter can also be expressed in terms of fractional derivative as

$$
Q_{d u}=V_{p} \rho_{f} C_{f} \frac{24}{\mathrm{Pe}_{p} \sqrt{\tau_{d u}}}\left(\frac{D^{1 / 2} T_{f}}{D t^{1 / 2}}-\frac{d^{1 / 2} T_{p}}{d t^{1 / 2}}\right),
$$

which is more convenient to estimate the ratio $Q_{d u} / Q_{q s}$. Similar fractional derivative form has been used for Basset history force in the work of Bagchi and Balachandar ${ }^{45}$ and Ling et al. ${ }^{14}$ Then $Q_{d u} / Q_{q s}$ can be written as

$$
\frac{Q_{d u}}{Q_{q s}}= \begin{cases}\beta_{\theta} C_{d u} I_{d u, n i n t}, & \text { if } \tau_{\eta}>\tau_{d u}, \\ \sqrt{12 \beta_{\theta} / \Psi} I_{d u, \text { int }}, & \text { if } \tau_{\eta}<\tau_{d u},\end{cases}
$$

where $I_{d u, n i n t}$ and $I_{d u \text {,int }}$ are correction functions corresponding to the non-integral and integral forms of $Q_{d u}$, defined as

$$
\begin{aligned}
I_{d u, \text { int }} & =\frac{\tau_{p \theta}\left|D T_{f} / D t-d T_{p} / d t\right|}{\left|T_{f}-T_{p}\right|}, \\
I_{d u, \text { int }} & =\frac{\sqrt{\tau_{p \theta}}\left|D^{1 / 2} T_{f} / D t^{1 / 2}-d^{1 / 2} T_{p} / d t^{1 / 2}\right|}{\left|T_{f}-T_{p}\right|} .
\end{aligned}
$$

It can be easily shown that $I_{d u, \text { nint }}=I_{d u, \text { int }}=0$ for $\tau_{l}>\tau_{p \theta}$, and $I_{d u, i n t}=I_{d u, n i n t} / \sqrt{\operatorname{St}_{\theta, l}}$ for $\tau_{l}<\tau_{p \theta}$. With Eqs. (44) and (45), $I_{d u, \text { int }}$ can be approximated as

$$
I_{d u, \text { nint }}= \begin{cases}I_{u u}, & \text { if } \tau_{l}<\tau_{p \theta}, \\ 0, & \text { if } \tau_{l}>\tau_{p \theta} .\end{cases}
$$

Therefore, $I_{d u, n i n t}=0$ for regime I particles, while for regime III particles, $I_{d u \text {,nint }}$ is identical to $I_{u u}$. In regime II, $I_{d u, n i n t}=0$ when $\tau_{l}>\tau_{p \theta}$ and $I_{d u \text {,nint }}=I_{u u}$ when $\tau_{l}<\tau_{p \theta}$. Schematics of $I_{d u \text {,nint }}$ and $I_{d u \text {,nint }}$ are shown in Fig. 5(b). It is seen that the relative importance of the diffusive-unsteady heat transfer is again the strongest for the Kolmogorov scale eddies. These estimates of the relative importance of different unsteady contributions to inter-phase heat transfer compared to the quasi-steady contribution are summarized in Table I, along with the corresponding results on

\begin{tabular}{|c|c|c|c|c|c|}
\hline \multicolumn{3}{|c|}{ Force } & \multicolumn{3}{|c|}{ Heat transfer } \\
\hline & Reg I & Reg II, III & & Reg I & Reg II, III \\
\hline$\left|\boldsymbol{F}_{s g} / \boldsymbol{F}_{q s}\right|$ & $\beta_{\rho} \frac{1}{\left|1-\beta_{\rho}\right|}$ & $\beta_{\rho} \frac{\tau_{p m} / \tau_{\eta}}{|1-\beta \rho|}$ & $\left|Q_{u u} / Q_{q s}\right|$ & $\beta_{\theta} \frac{1}{\left|1-\beta_{\theta}\right|}$ & $\beta_{\theta} \frac{\tau_{p \theta} / \tau_{\eta}}{\left|1-\beta_{\theta}\right|}$ \\
\hline$\left|\boldsymbol{F}_{a m} / \boldsymbol{F}_{q s}\right|$ & 0 & $\beta_{\rho} C_{M} \frac{\tau_{p m} / \tau_{\eta}}{|1-\beta \rho|}$ & & & \\
\hline$\left|\boldsymbol{F}_{v u, \text { nint }} / \boldsymbol{F}_{q s}\right|$ & 0 & $\beta_{\rho} C_{v u} \frac{\tau_{p m} / \tau_{\eta}}{|1-\beta \rho|}$ & $\left|Q_{d u, \text { nint }} / Q_{q s}\right|$ & 0 & $\beta_{\theta} C_{d u} \frac{\tau_{p \theta} / \tau_{\eta}}{\left|1-\beta_{\theta}\right|}$ \\
\hline$\left|\boldsymbol{F}_{v u, i n t} / \boldsymbol{F}_{q s}\right|$ & 0 & $\sqrt{\frac{3 \beta}{\pi \Phi}} \frac{\sqrt{\tau_{p m} / \tau_{\eta}}}{|1-\beta|}$ & $\left|Q_{d u, i n t} / Q_{q s}\right|$ & 0 & $\sqrt{\frac{12 \beta_{\theta}}{\Psi}} \frac{\sqrt{\tau_{p \theta} / \tau_{\eta}}}{\left|1-\beta_{\theta}\right|}$ \\
\hline
\end{tabular}
the relative importance of the unsteady forces. The special cases of heavy particle limit (i.e., $\beta_{\rho}=\rho_{f} / \rho_{p} \ll 1$ and $\beta_{\theta} \ll 1$ ) and light bubbles (i.e., $\beta_{\rho} \gg 1$ and $\beta_{\theta} \gg 1$ ) are given in Tables II and III.

TABLE I. Scaling estimates of relative importance of unsteady force and heat transfer comparing to quasi-steady force and heat transfer in turbulent multiphase flows, where $\beta_{\rho}=\rho_{f} / \rho_{p}, \beta_{\theta}=\left(\rho_{f} C_{f}\right) /\left(\rho_{p} C_{p}\right)$, and $\beta=3 /\left(2 \rho_{p} / \rho_{f}+1\right)$. 
TABLE II. Scaling estimates of relative importance of unsteady force and heat transfer comparing to quasi-steady force and heat transfer in turbulent multiphase flows in the limit of gas-particles flows, namely, $\beta_{\rho} \ll 1, \beta_{\theta} \ll$ 1 , and $\beta \rightarrow 3 / 2 \beta_{\rho}$.

\begin{tabular}{|c|c|c|c|c|c|}
\hline \multicolumn{3}{|c|}{ Force } & \multicolumn{3}{|c|}{ Heat transfer } \\
\hline & Reg I & Reg II, III & & Reg I & Reg II, III \\
\hline$\left|\boldsymbol{F}_{s g} / \boldsymbol{F}_{q s}\right|$ & $\beta_{\rho}$ & $\frac{1}{18 \Phi}\left(\frac{d_{p}}{\eta}\right)^{2}$ & $\left|Q_{u u} / Q_{q s}\right|$ & $\beta_{\theta}$ & $\frac{\operatorname{Pr}_{f}}{12 \Psi}\left(\frac{d_{p}}{\eta}\right)^{2}$ \\
\hline$\left|\boldsymbol{F}_{a m} / \boldsymbol{F}_{q s}\right|$ & 0 & $\frac{C_{M}}{18 \Phi}\left(\frac{d_{p}}{\eta}\right)^{2}$ & & & \\
\hline$\left|\boldsymbol{F}_{v u, \text { nint }} / \boldsymbol{F}_{q s}\right|$ & 0 & $\frac{C_{v u}}{18 \Phi}\left(\frac{d_{p}}{\eta}\right)^{2}$ & $\left|Q_{d u} / Q_{q s}\right|$ & 0 & $\frac{\operatorname{Pr}_{f} C_{d u}}{12 \Psi}\left(\frac{d_{p}}{\eta}\right)^{2}$ \\
\hline$\left|\boldsymbol{F}_{v u, \text { int }} / \boldsymbol{F}_{q s}\right|$ & 0 & $\frac{1}{2 \sqrt{\pi} \Phi} \frac{d_{p}}{\eta}$ & $\left|Q_{d u} / Q_{q s}\right|$ & 0 & $\frac{\sqrt{\operatorname{Pr}_{f}}}{\Psi}\left(\frac{d_{p}}{\eta}\right)$ \\
\hline
\end{tabular}

\section{Evaluate importance of unsteady heat transfer}

For regime I particles, $Q_{u u} / Q_{q s}=\beta_{\theta} /\left|1-\beta_{\theta}\right|$ and $Q_{d u} / Q_{q s}=0$. Therefore, when $\beta_{\theta}$ is small such as for gas-particle flows, the quasi-steady contribution always dominates inter-phase heat transfer and the unsteady heat transfer contributions can be ignored as has been done in many simulations of gas-particle flows. ${ }^{20,25}$

For regimes II and III particles, the maximum values of the ratios correspond to the Kolmogorov scale eddies. If a threshold value $\delta$ (say, for example, $\delta=5 \%$ ) is chosen, so that for values of $Q_{u u} / Q_{q s}$ and $Q_{d u} / Q_{q s}$ given in Eqs. (69) and (74) larger than the threshold, we will consider the corresponding unsteady contribution to heat transfer to the particle temperature evolution is significant when compared to the quasi-steady contribution.

With such a choice of threshold, the criteria for the importance of the undisturbed-unsteady heat transfer can be stated as

$$
\frac{\beta_{\theta} \mathrm{St}_{\theta, \eta}}{\left|1-\beta_{\theta}\right|}>\delta,
$$

and that for the diffusive-unsteady heat transfer can be expressed as

$$
\begin{cases}C_{d u} \frac{\beta_{\theta} \mathrm{St}_{\theta, \eta}}{\left|1-\beta_{\theta}\right|}>\delta, & \text { if } \tau_{\eta}>\tau_{d u}, \\ \sqrt{\frac{12}{\Psi}} \frac{\sqrt{\beta_{\theta} \mathrm{St}_{\theta, \eta}}}{\left|1-\beta_{\theta}\right|}>\delta, & \text { if } \tau_{\eta}<\tau_{d u} .\end{cases}
$$

It is seen that when $\tau_{\eta}>\tau_{d u}$, the criteria for $Q_{d u}$ are very similar to $Q_{u u}$. For gas-particle flows, $\beta_{\theta} \ll 1$ and thus $\left|1-\beta_{\theta}\right| \approx 1$. In such a case, Eqs. (78) and (79) can be further simplified as

TABLE III. Scaling estimates of relative importance of unsteady force and heat transfer comparing to quasi-steady force and heat transfer in turbulent multiphase flows in the limit of bubbly flows, namely, $\beta_{\rho} \gg 1, \beta_{\theta} \gg 1$, and

\begin{tabular}{|c|c|c|c|c|c|}
\hline \multicolumn{3}{|c|}{ Force } & \multicolumn{3}{|c|}{ Heat transfer } \\
\hline & Reg I & Reg II, III & & Reg I & Reg II, III \\
\hline$\left|\boldsymbol{F}_{s g} / \boldsymbol{F}_{q s}\right|$ & 1 & $\frac{1}{36 \Phi}\left(\frac{d_{p}}{\eta}\right)^{2}$ & $\left|Q_{u u} / Q_{q s}\right|$ & 1 & 1 \\
\hline$\left|\boldsymbol{F}_{a m} / \boldsymbol{F}_{q s}\right|$ & 0 & $\frac{C_{M}}{36 \Phi}\left(\frac{d_{p}}{\eta}\right)^{2}$ & & & \\
\hline$\left|\boldsymbol{F}_{v u, \text { nint }} / \boldsymbol{F}_{q s}\right|$ & 0 & $\frac{C_{v u}}{36 \Phi}\left(\frac{d_{p}}{\eta}\right)^{2}$ & $\left|Q_{d u} / Q_{q s}\right|$ & 0 & $C_{d u}$ \\
\hline$\left|\boldsymbol{F}_{v u, i n t} / \boldsymbol{F}_{q s}\right|$ & 0 & $\frac{1}{4 \sqrt{\pi} \Phi} \frac{d_{p}}{\eta}$ & $\left|Q_{d u} / Q_{q s}\right|$ & 0 & $\sqrt{\frac{12}{\Psi}}$ \\
\hline
\end{tabular}
$\beta \rightarrow 3$. 


$$
\frac{d_{p}}{\eta}>\sqrt{\frac{12 \Psi \delta}{\operatorname{Pr}_{f}}}
$$

and

$$
\begin{cases}\frac{d_{p}}{\eta}>\sqrt{\frac{12 \Psi \delta}{\operatorname{Pr}_{f} C_{d u}}}, & \text { if } \tau_{\eta}>\tau_{d u}, \\ \frac{d_{p}}{\eta}>\frac{\Psi \delta}{\sqrt{\operatorname{Pr}_{f}}}, & \text { if } \tau_{\eta}<\tau_{d u} .\end{cases}
$$

It is clearly shown that for gas-particle flows, the importance of both undisturbed-unsteady and diffusive-unsteady heat transfer actually depends on particle-to-fluid length scale ratio, i.e., the ratio between the particle size and the Kolmogorov length scale, instead of the volumetric heat capacity ratios.

The above criteria are derived based on particle's response to Kolmogorov scale eddies. If the above conditions (Eqs. (78)-(81)) are satisfied, it implies that a range of eddies of size $\eta$ and larger influence the particle temperature evolution not only through the quasi-steady contribution but also through the undisturbed-unsteady and diffusive-unsteady contributions of heat transfer. This importance of unsteady heat transfer may only be limited to the influence of the small scale turbulent eddies. Nevertheless, in the context of direct numerical simulation (DNS), where all the scales of turbulent motion are fully resolved, it is essential to account for the influence of the entire range of flow scales on particle temperature evolution.

The above argument can be easily extended to large eddy simulations (LES), where a cut-off length scale $l_{c}$ is defined, and only eddies larger than $l_{c}$ are directly computed. In such a case, the maximum values of the ratios between the unsteady and quasi-steady contributions to heat transfer are dictated by the smallest resolved eddies of length scale $l_{c}$, as shown in Fig. 5. As a result, the importance of unsteady heat transfer for gas-particle flows arising from the resolved scales can be again evaluated by Eqs. (80) and (81), except with $\eta$ replaced by $l_{c}$. At last, it should be reminded when the particle is small compared to the LES cut-off length scale but is larger or comparable to the Kolmogorov length scale, then the unsteady heat transfer due to the resolved scales may be small, but that arising from the unresolved scales can be significant and thus needs to be taken into account in subgrid closure model.

\section{B. Importance of inter-phase heat transfer in backward energy coupling}

In the work of Crowe et al. ${ }^{17}$ and Ling et al., ${ }^{14}$ the momentum coupling parameter has been used to assess the importance of different forces in backward momentum coupling. The momentum coupling parameter is defined as the ratio between the backward coupling force contribution and the inertial term in the fluid momentum equation. Similarly, to assess the importance of the different contributions to backward energy coupling, an energy coupling parameter can be defined here as the ratio between the backward coupling contribution and the thermal inertial term in the fluid internal energy equation (Eq. (36)). When the energy coupling parameter is large, such as $\sim O(1)$, the two-way coupling effect of the corresponding contribution to fluid internal energy equation is significant.

\section{Quasi-steady heat transfer}

The energy coupling parameter for the quasi-steady heat transfer can be expressed as

$$
\Gamma_{q q s}=\frac{\left|\rho_{p} \phi_{p} q_{q s}\right|}{\left|\rho_{f} \phi_{f} \frac{D e_{f}}{D t}\right|} .
$$

With the scaling estimates obtained in Eqs. (48)-(55), $\Gamma_{q q s}$ can be written as

$$
\Gamma_{q q s} \approx \frac{Y_{p} C_{p}}{Y_{f} C_{f}}\left|1-\beta_{\theta}\right| J_{q q s},
$$

where the correction function $J_{q q s}=1 / I_{u u}$ (see Eq. (70)) and can be estimated in different regimes as 


$$
J_{q q s} \approx \begin{cases}1, & \text { regime I, } \\ \frac{1}{\mathrm{St}_{\theta, L}}, & \text { regimes II and III. }\end{cases}
$$

For gas-particle flows $\left(\beta_{\theta} \ll 1\right)$, Eq. (83) becomes

$$
\Gamma_{q q s} \approx \frac{Y_{p} C_{p}}{Y_{f} C_{f}} J_{q q s}
$$

For small particles $J_{q q s}=1$, and as a result, the particle-to-fluid mass fraction ratio $Y_{p} / Y_{f}$ multiplied by the specific heat ratio $C_{p} / C_{f}$ is sufficient to evaluate the importance of the contribution of quasi-steady heat transfer in the backward energy coupling. Attention is required for large particles as the correction function $J_{q q s}=1 / \mathrm{St}_{\theta, L}$ can be quite large. Similar observations have been made on momentum coupling parameter by Ling et al. ${ }^{14}$

For bubbly flows $\left(\beta_{\theta} \gg 1\right)$, Eq. (83) becomes

$$
\Gamma_{q q s} \approx \frac{\phi_{p}}{\phi_{f}} J_{q q s} .
$$

Then it is clearly seen that the importance of quasi-steady heat transfer in backward energy coupling depends on the particle-to-fluid volume fraction ratio instead of mass fraction ratio.

\section{Undisturbed-unsteady heat transfer}

Similarly, we can estimate the energy coupling parameter for the undisturbed-steady heat transfer as

$$
\Gamma_{q u u} \approx \frac{\left|\rho_{p} \phi_{p} q_{u u}\right|}{\left|\rho_{f} \phi_{f} \frac{D e_{f}}{D t}\right|} \approx \frac{\phi_{p}}{\phi_{f}} .
$$

It is seen that the energy coupling parameter is independent of volumetric heat capacity ratio and depends on the particle-to-fluid volume fraction ratio.

\section{Diffusive-unsteady heat transfer}

The energy coupling parameter corresponding to diffusive-unsteady heat transfer is defined as

$$
\Gamma_{q d u} \approx \frac{\left|\rho_{p} \phi_{p} q_{d u}\right|}{\left|\rho_{f} \phi_{f} \frac{D e_{f}}{D t}\right|} .
$$

Depending on the parameter $\tau_{\eta} / \tau_{d u}, \Gamma_{q d u}$ can be estimated based on either the non-integral (Eq. (62)) or integral (Eq. (15)) expressions. It can shown that

$$
\Gamma_{q d u} \approx \begin{cases}0, & \text { Regime I, } \\ \frac{\phi_{p}}{\phi_{f}} J_{q d u}, & \text { Regime II and III. }\end{cases}
$$

where the correction function $J_{q d u}$ can be estimated in different regimes as

$$
J_{q d u} \approx \begin{cases}C_{d u}, & \text { if } \tau_{\eta}>\tau_{d u}, \\ 12 \frac{\eta}{d_{p}} \operatorname{Re}_{L}^{1 / 4}, & \text { if } \tau_{\eta}<\tau_{d u},\end{cases}
$$

where $\operatorname{Re}_{L}=u_{L} L / v_{f}$ is the Reynolds number based on the integral length scale. Since regime I particles respond to eddies of all scales, the diffusive-unsteady contribution of heat transfer is very small (identical to zero based on the present scaling argument); therefore, its contribution to continuous phase energy equation is also negligible. For regimes II and III particles, the energy coupling parameter for diffusive-unsteady heat transfer depends on the particle-to-fluid volume fraction ratio, similar to undisturbed-unsteady heat transfer. Depending on the particle diffusive-unsteady time scale $\tau_{d u}$ in relation to the Kolmogorov time scale $\tau_{\eta}$, two different estimates are obtained for the correction function $J_{q d u}$. It is noted that $J_{q d u}$ can be much larger than one in either situation given in Eq. (90). 
As an example, for solid particles of size comparable to the Kolmogorov length scale in a turbulent gas flow with $\operatorname{Re}_{L}=1000$ and $\operatorname{Pr}_{f}=0.7$, it can be shown that $J_{q d u} \approx 3.05$ and 67.5 from Eq. (90). Therefore, $J_{q d u}$ must be taken into account in estimating the importance of diffusive-unsteady heat transfer in backward energy coupling for particles in regimes II and III.

\section{Importance of work done by inter-phase coupling forces in backward energy coupling}

Similar to the inter-phase heat transfer, the importance of the work done by the quasi-steady and viscous-unsteady forces in backward energy coupling can be assessed by the corresponding energy coupling parameters.

\section{Quasi-steady force}

The energy coupling parameter for the quasi-steady force can be expressed as

$$
\Gamma_{f q s}=\frac{\left|\rho_{p} \phi_{p} \mathbf{f}_{q s} \cdot\left(\mathbf{u}_{f}-\mathbf{u}_{p}\right)\right|}{\left|\rho_{f} \phi_{f} \frac{D e_{f}}{D t}\right|} .
$$

With the scaling estimates given in Eqs. (48)-(55), $\Gamma_{f q s}$ in the three regimes can be approximated as

$$
\Gamma_{f q s} \approx \frac{Y_{p}}{Y_{f}} \operatorname{Ec}\left(1+\frac{\rho_{f}}{2 \rho_{p}}\right)|1-\beta|^{2} J_{f q s},
$$

where the correction function $J_{f q s}$ can be expressed as

$$
J_{f q s} \approx \begin{cases}\mathrm{St}_{\theta, \eta}, & \text { regime I, } \\ \max \left(\mathrm{St}_{\theta, \eta}, \frac{1}{\mathrm{St}_{\theta, L}}\right), & \text { regime II, } \\ \frac{1}{\mathrm{St}_{\theta, L}}, & \text { regime III. }\end{cases}
$$

Here the Eckert number is defined as Ec $=u_{l}^{2} /\left(C_{f} T_{l}\right)$, and for simplicity is considered a constant, independent of the eddy size $l$. For gas-particle flows, Eq. (92) becomes

$$
\Gamma_{f q s} \approx \frac{Y_{p}}{Y_{f}} \operatorname{Ec} J_{f q s}
$$

while for bubbly flows $\left(\rho_{f} \gg \rho_{p}\right)$, Eq. (92) becomes

$$
\Gamma_{f q s} \approx 2 \frac{\phi_{p}}{\phi_{f}} \mathrm{Ec} J_{f q s} .
$$

It is shown that the energy coupling parameter for the quasi-steady force is proportional to particleto-fluid mass fraction ratio $Y_{p} / Y_{f}$ for heavy particles and to particle-to-fluid volume fraction ratio $\phi_{p} / \phi_{f}$ for bubbles. This is consistent with the observations of the momentum coupling parameter corresponding to the quasi-steady force.

It can be also seen that $\Gamma_{f q s}$ is related to the Eckert number. Generally, Ec is very small in incompressible turbulent flows. Therefore, except for special cases where $Y_{p} / Y_{f}, \phi_{p} / \phi_{f}$, or $J_{f q s}$ are very large, $\Gamma_{f q s}$ is generally quite small. Furthermore, it can be shown from Eqs. (85) and (94) (or Eqs. (86) and (95)) that $\Gamma_{f q s} / \Gamma_{q q s}$ is proportional to Ec for both gas-particle and bubbly flows. Therefore, when $\mathrm{Ec}$ is small, the contribution of work done by the quasi-steady force on backward energy coupling is generally less important than the quasi-steady heat transfer.

\section{Viscous-unsteady force}

The energy coupling parameter for the viscous-unsteady force is given as

$$
\Gamma_{f v u}=\frac{\left|\rho_{p} \phi_{p} \mathbf{f}_{v u} \cdot\left(\mathbf{u}_{f}-\mathbf{u}_{p}\right)\right|}{\left|\rho_{f} \phi_{f} \frac{D e_{f}}{D t}\right|}
$$


and can be estimated in the three regimes as

$$
\Gamma_{f v u} \approx \begin{cases}0, & \text { regime I, } \\ \frac{\phi_{f}}{\phi_{p}} J_{f v u}, & \text { regimes II and III, }\end{cases}
$$

where $J_{f v u}$ is a correction function given as

$$
J_{f v u}=\frac{9 \mathrm{Ec}}{\sqrt{\pi}} \frac{\eta}{d_{p}} \operatorname{Re}_{L}^{1 / 4} .
$$

It is seen that, similar to $\Gamma_{q d u}, \Gamma_{f v u}$ is proportional to the particle-to-fluid volume fraction ratio instead of mass fraction ratio for all $\rho_{p} / \rho_{f}$. Again it can be shown from Eqs. (89) and (97) that $\Gamma_{f v u} / \Gamma_{q d u}$ is proportional to Ec. As a result, the contribution of the work done by the viscous-unsteady force in changing the fluid temperature is generally small compared to that of the diffusive-unsteady heat transfer.

Finally, it is noted that the above scaling approach in estimating the importance of inter-phase heat transfer and the work done by the coupling forces on the fluid internal energy evolution can also be applied to other terms of Eq. (36). For example, the ratio between the viscous dissipation and the thermal inertia term can be estimated as $\left(\boldsymbol{\tau}_{f}: \nabla \mathbf{u}_{m}\right) /\left(\rho_{f} \phi_{f} D e_{f} / D t\right) \approx \mathrm{Ec} / \mathrm{Re}$. As expected the contribution of viscous dissipation to the fluid internal energy is not significant since Ec is small in general.

\section{CONCLUSIONS}

Modeling of inter-phase momentum and energy coupling is critical to accurate simulation of turbulent multiphase flows. In this paper, we address four important fundamental questions about inter-phase energy coupling in turbulent multiphase flows: (1) What is the contribution of the interphase coupling forces to inter-phase energy coupling? (2) When and how can we simplify the integral representation of diffusive-unsteady heat transfer? (3) Under what conditions unsteady heat transfer is important to the energy balance of the particles? (4) What parameters accurately evaluate the importance of back effect of both the inter-phase heat transfer and coupling forces in the fluid energy equation? The key findings of the present study in regard to these four questions are summarized separately as follows.

\section{A. Conclusions on the contribution of the inter-phase coupling forces to energy coupling}

By Newton's third law, the inter-phase force components on the fluid and particle phases are opposite of each other. However, since the macroscale fluid and particle phase velocities are different (i.e., $\mathbf{u}_{f} \neq \mathbf{u}_{p}$ ), the kinetic energy contribution of the inter-phase coupling forces to the macroscale fluid and particle motion will not balance. The difference between the two must be balanced by changes to the kinetic energy of the microscale motion of the fluid around the particles. Due to the viscous origin of the quasi-steady force, its contribution to microscale kinetic energy is dissipated and transferred to the internal energy of the fluid. However, due to the inviscid origin of the added-mass force, its contribution to the microscale kinetic energy cannot contribute to dissipative transfer to internal energy, as was done for the quasi-steady force. The microscale kinetic energy transfer due to viscous-unsteady force is more complicated. It is partly dissipative like the quasi-steady force, and partially non-dissipative and used to modify the near-field viscous microscale flow structure in the vicinity of the particle. It is difficult to separate the two contributions in the time domain, but over long time, the dissipation portion can be expected to dominate.

\section{B. Conclusions on representation of diffusive-unsteady heat transfer}

In general, the diffusive-unsteady contribution to heat transfer is given by a Basset-like convolution integral, whose computation is often costly. However, when both the particle and the fluid acceleration are sufficiently slow, it is possible to simplify the conventional formulation and avoid 
evaluating the convolution integral as in Eq. (62). Here we establish the condition under which such a simplification can be made. The main conclusions are as follows:

- Only when the diffusive-unsteady time scale $\tau_{d u}$ (defined as the transition time for the kernel to change from the slow $t^{-1 / 2}$ decay to the fast exponential decay) is smaller than all the fluid time scales, the diffusive-unsteady heat transfer formulation can be simplified. As $\tau_{\eta}$ is the smallest fluid time scale, the time scale ratio $\tau_{\eta} / \tau_{d u}$ can be used to determine when the simplified formulation (Eq. (62)) can be used.

- In turbulent multiphase flows, $\tau_{\eta} / \tau_{d u}$ increases monotonically with $d_{p} / \eta$. Therefore, there exists a critical diameter $d_{p, d u}^{*}$ and when particle size is smaller (i.e., $d_{p}<d_{p, d u}^{*}$ ), the diffusiveunsteady heat transfer needs to be calculated using the convolution history integral as Eq. (15), while for $d_{p}>d_{p, d u}^{*}$, the simplified non-integral form, Eq. (62), can be used.

\section{Conclusions on the importance of unsteady heat transfer in forward energy coupling}

The ratio between the magnitudes of unsteady and quasi-steady contributions of heat transfer is used to evaluate the importance of the unsteady heat transfer in the energy equation of the particles. Scaling estimates of the ratios are obtained and the key conclusions include the following:

- Unsteady heat transfer is important in evaluating the thermal evolution of particles when the fluid-to-particle volumetric heat capacity ratio is large, i.e., $\beta_{\theta} \gtrsim 1$.

- Conventionally, unsteady contributions to heat transfer are neglected in gas-particle flows based on the assumption that these contributions are small compared to quasi-steady heat transfer as the fluid-to-particle volumetric heat capacity ratio is small. However, the present scaling analysis shows that for gas-particle flows $\left(\rho_{p} C_{p} \gg \rho_{f} C_{f}\right)$ the importance of the unsteady heat transfer depends on the ratio between the particle size and the Kolmogorov length scale and not on the particle-to-fluid volumetric heat capacity ratio.

- In DNS of turbulent multiphase flows, the intent is to accurately compute all the turbulent length scales and their influence on particle motion and thermal evolution. When the particle size is comparable or larger than the Kolmogorov scale (i.e., smallest fluid length scale), unsteady heat transfer due to the smallest eddies is important in accurately evaluating the particle temperature.

\section{Conclusions on the importance of inter-phase heat transfer and work done by inter-phase forces in the energy balance of the fluid}

We define the energy coupling parameter as the ratio between the contribution of inter-phase heat transfer (or work done by the inter-phase coupling forces) to the fluid thermal inertia term, which serves as a measure of their relative importance in backward energy coupling. The key conclusions of the scaling argument are as follows:

- It is shown that the energy coupling parameter of quasi-steady heat transfer depends on $Y_{p} C_{p} /$ $\left(Y_{f} C_{f}\right)$ and $\phi_{p} / \phi_{f}$ for gas-particle and bubbly flows, respectively. The energy coupling parameter is also modified by a correction function $J_{q q s}$, which is equal to $1 / \mathrm{St}_{\theta, L}$ for particles with thermal time scale larger than the Kolmogorov time scale. The correction function can be larger than unity when the particle thermal time scale is larger than the turbulence integral time scale $\left(\tau_{p \theta}>\tau_{L}\right)$.

- The energy coupling parameters of undisturbed-unsteady and diffusive-unsteady heat transfer are similar, which are related to the particle volume fraction. Similar to the quasi-steady heat transfer, the energy coupling parameter of the diffusive-unsteady heat transfer is also related to a correction function, which can be larger than unity for particles with $\tau_{p \theta}>\tau_{L}$.

- For gas-particle flows, the energy coupling parameters corresponding to the work done by the quasi-steady and viscous-unsteady forces are related to the particle-to-fluid mass fraction and volume fraction ratios, respectively. The energy coupling parameters of the quasi-steady $\left(\Gamma_{f q s}\right)$ and viscous-unsteady forces $\left(\Gamma_{f v u}\right)$ are similar to those of the quasi-steady $\left(\Gamma_{q q s}\right)$ and diffusiveunsteady heat transfer $\left(\Gamma_{q d u}\right)$, respectively. The ratios between $\Gamma_{f q s}$ and $\Gamma_{q q s}$ and between $\Gamma_{f v u}$ and $\Gamma_{q d u}$ are both proportional to the Eckert number. 


\section{ACKNOWLEDGMENTS}

This work was supported by the U.S. Department of Energy, National Nuclear Security Administration, Advanced Simulation and Computing Program, as a Cooperative Agreement under the Predictive Science Academic Alliance Program, under Contract No. DE-NA0002378, and by the Defense Threat Reduction Agency, Basic Research Award No. HDTRA1-14-1-0028 to University of Florida, Gainesville, U.S. This work has also been supported by the Agence nationale de la recherche in France, under the MODEMI Project No. ANR-11-MONU-0011.

${ }^{1}$ M. R. Maxey and J. J. Riley, "Equation of motion for a small rigid sphere in a nonuniform flow," Phys. Fluids 26, 883-889 (1983).

${ }^{2}$ R. Gatignol, "The Faxén formulae for a rigid particle in an unsteady non-uniform Stokes flow," J. Mec. Theor. Appl. 2, $143-160$ (1983).

${ }^{3}$ M. Parmar, A. Haselbacher, and S. Balachandar, "Generalized Basset-Boussinesq-Oseen equation for unsteady forces on a sphere in a compressible flow,” Phys. Rev. Lett. 106, 084501 (2011).

${ }^{4}$ M. Parmar, A. Haselbacher, and S. Balachandar, "Equation of motion for a sphere in equation of motion for a sphere in non-uniform compressible flows," J. Fluid Mech. 699, 352-375 (2012).

${ }^{5}$ R. Mei and R. J. Adrian, "Flow past a sphere with an oscillation in the free-stream velocity and unsteady drag at finite Reynolds number," J. Fluid Mech. 237, 323-341 (1992).

${ }^{6}$ J. Magnaudet and I. Eames, “The motion of high-Reynolds-number bubbles in inhomogeneous flows,” Annu. Rev. Fluid Mech. 32, 659-708 (2000).

${ }^{7}$ S. Balachandar, "A scaling analysis for point particle approaches to turbulent multiphase flows," Int. J. Multiphase Flow 35, 801-810 (2009).

${ }^{8}$ S. Balachandar and J. K. Eaton, "Turbulent dispersed multiphase flow," Annu. Rev. Fluid Mech. 42, 111-133 (2010).

${ }^{9}$ Y. Ling, A. Haselbacher, S. Balachandar, F. M. Najjar, and D. S. Stewart, "Shock interaction with a deformable particle: Direct numerical simulations and point-particle modeling," J. Appl. Phys. 113, 013504 (2013).

${ }^{10}$ E. E. Michaelides and Z. Feng, "Heat transfer from a rigid sphere in a nonuniform flow and temperature field," Int. J. Heat Mass Transfer 37, 2069-2076 (1994).

${ }^{11}$ Z. G. Feng and E. E. Michaelides, "Unsteady heat transfer from a sphere at small peclet numbers," J. Fluids Eng. 118, 96-102 (1996).

${ }^{12}$ S. Balachandar and M. Y. Ha, "Unsteady heat transfer from a sphere in a uniform cross-flow," Phys. Fluids 13, 3714-3728 (2001).

${ }^{13}$ Y. Ling, J. L. Wagner, S. J. Beresh, S. P. Kearney, and S. Balachandar, "Interaction of a planar shock wave with a dense particle curtain: Modeling and experiments," Phys. Fluids 24, 113301 (2012).

${ }^{14}$ Y. Ling, M. Parmar, and S. Balachandar, "A scaling analysis of added-mass and history forces and their coupling in dispersed multiphase flows," Int. J. Multiphase Flow 57, 102-114 (2013).

${ }^{15} \mathrm{Y}$. Xu and S. Subramaniam, "Consistent modeling of interphase turbulent kinetic energy transfer in particle-laden turbulent flows," Phys. Fluids 19, 085101 (2007).

${ }^{16}$ M. Mehrabadi, S. Tenneti, R. Garg, and S. Subramaniam, "Pseudo-turbulent gas-phase velocity fluctuations in homogeneous gas-solid flow: Fixed particle assemblies and freely evolving suspensions," J. Fluid Mech. 770, 210-246 (2015).

${ }^{17}$ C. T. Crowe, M. Sommerfield, and Y. Tsuji, Multiphase Flows with Droplets and Particles (CRC Press, 1998).

${ }^{18}$ S. Corrsin, "On the spectrum of isotropic temperature fluctuations in an isotropic turbulence," J. Appl. Phys. 22, 469-473 (1951).

${ }^{19}$ M. Piller, E. Nobile, and T. J. Hanratty, "DNS study of turbulent transport at low Prandtl numbers in a channel flow," J. Fluid Mech. 458, 419-441 (2002).

${ }^{20}$ Y. Ling, A. Haselbacher, and S. Balachandar, "Transient phenomena in one-dimensional compressible gas-particle flows," Shock Waves 19, 67-81 (2009).

${ }^{21}$ K. D. Squires and J. K. Eaton, "Preferential concentration of particles by turbulence," Phys. Fluids A 3, 1169-1178 (1991).

${ }^{22}$ S. Elghobashi and G. C. Truesdell, "Direction simulation of particle dispersion in a decaying isotropic turbulence," J. Fluid Mech. 242, 655-700 (1992).

${ }^{23}$ M. J. Andrews and P. J. O'Rourke, “The multiphase particle-in-cell (MP-PIC) method for dense particulate flows," Int. J. Multiphase Flow 22, 379-402 (1996).

${ }^{24}$ D. M. Snider, P. J. O'Rourke, and M. J. Andrews, "Sediment flow in inclined vessels calculated using a multiphase particle-in-cell model for dense particle flows," Int. J. Multiphase Flow 24, 1359-1382 (1998).

${ }^{25}$ K. Balakrishnan, D. V. Nance, and S. Menon, "Simulation of impulse effects from explosive charges containing metal particles," Shock Waves 20, 217-239 (2010).

${ }^{26}$ Y. Ling, A. Haselbacher, and S. Balachandar, "Importance of unsteady contributions to force and heating for particles in compressible flows. Part 2: Application to particle dispersal by blast wave," Int. J. Multiphase Flow 37, 1013-1025 (2011).

${ }^{27}$ R. Garg, C. Narayanan, D. Lakehal, and S. Subramaniam, "Accurate numerical estimation of interphase momentum transfer in Lagrangian-Eulerian simulations of dispersed two-phase flows," Int. J. Multiphase Flow 33, 1337-1364 (2007).

${ }^{28}$ L. Schiller and Z. Naumann, “Über die grundlegenden Kräfte bei der schwerkraftaufbereitung," Z. Ver. Dtsch. Ing. 77, 318-320 (1933).

${ }^{29}$ R. Clift and W. H. Gauvin, “The motion of particles in turbulent gas streams," Proc. Chemeca 1, 14-28 (1970).

${ }^{30}$ Y. Ling, A. Haselbacher, and S. Balachandar, "Importance of unsteady contributions to force and heating for particles in compressible flows. Part 1: Modeling and analysis for shock-particle interaction,” Int. J. Multiphase Flow 37, 1026-1044 (2011). 
${ }^{31} \mathrm{~S}$. Whitaker, "Forced convection heat transfer correlations for flow in pipes, past flat plates, single spheres, and for flow in packed beds and tube bundles," AIChE J. 18, 361-371 (1972).

${ }^{32}$ W. E. Ranz and W. R. Marshall, "Evaporation from droplets: Part 1," Chem. Eng. Prog. 48, 141-146 (1952).

${ }^{33}$ Computational Methods for Multiphase Flow, edited by A. Prosperetti and G. Tryggvason (Cambridge University Press, 2007).

${ }^{34}$ R. Saurel and R. Abgrall, "A multiphase Godunov method for compressible multifluid and multiphase flows," J. Comput. Phys. 150, 425-467 (1999).

${ }^{35}$ D. Z. Zhang and A. Prosperetti, "Averaged equations for inviscid disperse two-phase flow," J. Fluid Mech. 267, 185-220 (1994).

${ }^{36}$ M. Parmar, A. Haselbacher, and S. Balachandar, "On the unsteady inviscid force on cylinders and spheres in subcritical compressible flow," Philos. Trans. R. Soc., A 366, 2161-2175 (2008).

${ }^{37}$ M. Parmar, S. Balachandar, and A. Haselbacher, "Equation of motion for a drop or bubble in viscous compressible flows," Phys. Fluids 24, 056103 (2012).

${ }^{38}$ G. K. Batchelor, "Small-scale variation of convected quantities like temperature in turbulent fluid Part I. General discussion and the case of small conductivity," J. Fluid Mech. 5, 113-133 (1959).

${ }^{39}$ J. Mohd-Yusof, "Interaction of massive particles with turbulence," Ph.D. thesis, Cornell University, 1996.

${ }^{40}$ M. G. Pai and S. Subramaniam, "Modeling interphase turbulent kinetic energy transfer in Lagrangian-Eulerian spray computations," Atomization Sprays 16, 807-826 (2006).

${ }^{41}$ Y. Xu and S. Subramaniam, "A multiscale model for dilute turbulent gas-particle flows based on the equilibration of energy concept," Phys. Fluids 18, 033301 (2006).

${ }^{42}$ M. G. Pai and S. Subramaniam, "Modeling droplet dispersion and interphase turbulent kinetic energy transfer using a new dual-timescale Langevin model," Int. J. Multiphase Flow 33, 252-281 (2007).

43 J. Ferry and S. Balachandar, "Equilibrium Eulerian approach for predicting the thermal field of a dispersion of small particles," Int. J. Heat Mass Transfer 48, 681-689 (2005).

${ }^{44}$ A. Berrouk, D. Laurence, J. Riley, and D. Stock, "Stochastic modelling of inertial particle dispersion by subgrid motion for les of high Reynolds number pipe flow," J. Turbul. 8, N50 (2007).

${ }^{45}$ P. Bagchi and S. Balachandar, "Steady planar straining flow past a rigid sphere at moderate Reynolds number," J. Fluid Mech. 466, 365-407 (2002). 